PAJAR 2018 volume 6 (supplement 1) pages 1-48

http://revistaseletronicas.pucrs.br/ojs/index.php/pajar/

Pontifical Catholic University of Rio Grande do Sul

Institute of Geriatrics and Gerontology

Biomedical Gerontology Graduate Program

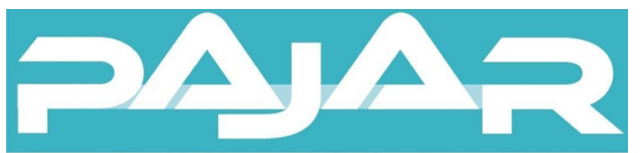

Pan American Journal of Aging Research

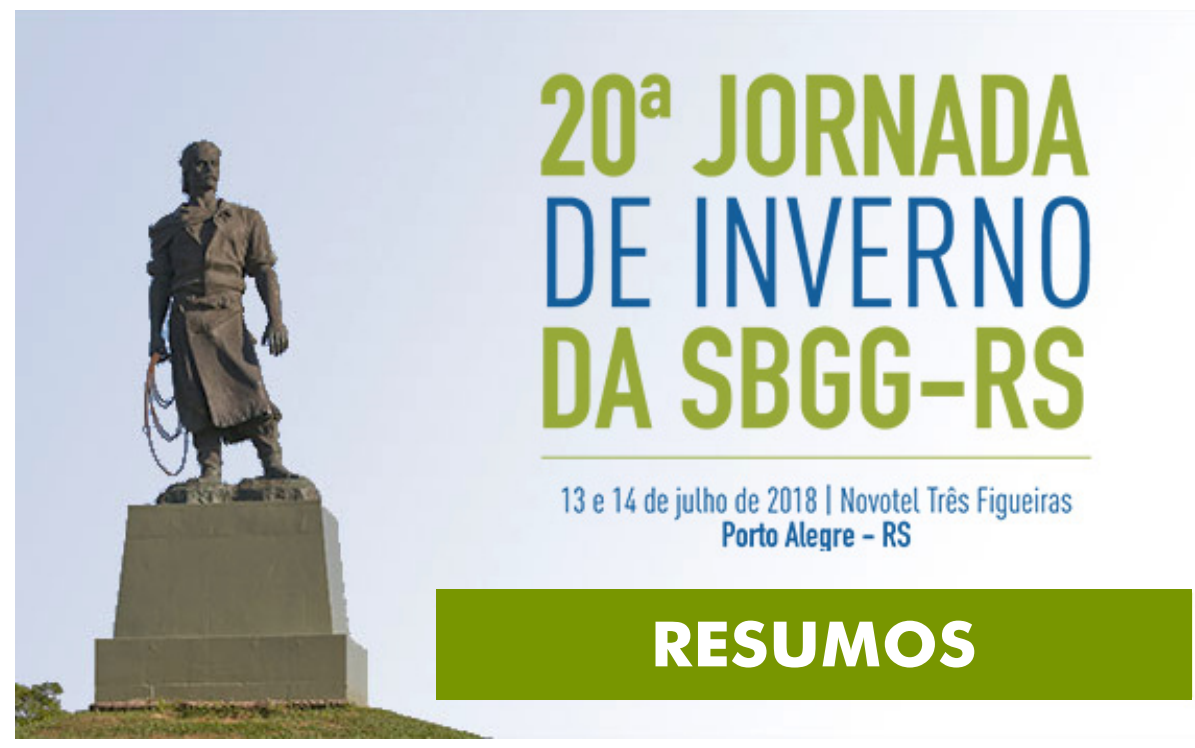




\title{
20 a Jornada de Inverno da Sociedade Brasileira de Geriatria e Gerontologia do Rio Grande do Sul (SBGG-RS)
}

\begin{abstract}
PAJAR (Pan American Journal of Aging Research) apresenta à comunidade Lcientífica o seu primeiro suplemento de 2018: Resumos da 20aㅡ Jornada de Inverno da SBGG-RS realizada na cidade de Porto Alegre/RS no período de 13 a 14 de julho de 2018.

Os resumos apresentados nesta edição foram selecionados do total de resumos do evento por se destacarem por sua qualidade científica e apresentação.

A PAJAR contribui para a divulgação de trabalhos científicos apresentados em eventos em busca permanente da qualificação de profissionais voltados a prática da saúde da população idosa.
\end{abstract}

Porto Alegre, 05 de novembro de 2018.

Alfredo Cataldo Neto Editor

Paula Engroff Editor Adjunto Alceu Valentino Panini Anelise Crippa Eunice Neves de Assis

Fernanda Loureiro

Guilherme Nogueira Editores Executivos 


\title{
Perfil clínico funcional de nonagenários com e sem incontinência urinária - dados do Projeto AMPAL
}

\author{
Vivian Ulrich', Josemara de Paula Rocha', Ângelo José Gonçalves Bós' \\ 'Instituto de Geriatria e Gerontologia, Pontifícia Universidade Católica do Rio Grande do Sul (IGG/PUCRS). < viviulrich@gmail.com> (correspondente)
}

INTRODUÇÃO: Segundo a Sociedade Internacional de Continência, a incontinência urinária (IU) é definida como "queixa de qualquer perda involuntária de urina" e é considerada, pela Organização Mundial de Saúde, parte das grandes síndromes geriátricas. A IU acarreta altos custos, impactos negativos na saúde e na qualidade de vida. OBJETIVO: Verificar o perfil clínico funcional de nonagenários e centenários com IU. MÉTODOS: Trata-se de um estudo transversal, realizado entre junho e dezembro de 2016, por meio de entrevista domiciliar com formulário padronizado. Foram analisados os idosos que responderam à questão "você às vezes perde urina mesmo que pouca (uma gota)?". Verificou-se a dificuldade para a realização das atividades de vida diária, participação em atividades sociais e sintomas sugestivos de depressão. RESULTADOS: Foram avaliados 192 longevos, sendo $134 \mathrm{com} \mathrm{IU} \mathrm{(70 \% ).} \mathrm{Estes} \mathrm{apresentaram} \mathrm{idade} \mathrm{semelhante,} \mathrm{mas} \mathrm{com} \mathrm{maior} \mathrm{prevalência} \mathrm{entre} \mathrm{as} \mathrm{mulheres}(n=110,82 \%)$. Observou-se que quanto maior a facilidade para realizar as atividades de vida diária, menor a perda urinária. A IU é mais incapacitante nas atividades de tomar banho sozinho $(p=0,00003)$, usar o banheiro $(p=0,00001)$, participar de atividades sociais $(p=0,08970)$. O longevo com IU sai de casa, em média, quatro vezes a menos por semana, comparado com quem não tem IU ( $p=0,02463)$. A presença de IU elevou em 30\% os sintomas sugestivos de depressão em longevos incontinentes $(p=0,05229)$. CONCLUSÕES: Longevos com perda urinária têm pior funcionalidade, saem menos de casa, participam menos de atividades sociais e apresentam mais sintomas sugestivos de depressão. Esses dados mostram a necessidade de medidas de prevenção ou tratamento da IU para uma longevidade com mais qualidade. 


\title{
Associação do Questionário SARC-F a circunferência da panturrilha como uma ferramenta de triagem da sarcopenia em uma amostra de idosas da região Noroeste do Rio Grande do Sul
}

\author{
Letícia Mazocco', Maria Cristina Gonzalez², Thiago G. Barbosa-Silva ${ }^{3}$, \\ Carla H. A. Schwanke' ${ }^{1}$ Patrícia Chagas ${ }^{4}$

\footnotetext{
'Programa de Pós-graduação em Gerontologia Biomédica, Escola de Medicina, Pontifícia Universidade Católica do Rio Grande do Sul (PUCRS). Porto Alegre, RS, Brasil.

2 Programa de Pós-Graduação em Saúde e Comportamento da Universidade Católica de Pelotas (UCPEL). Pelotas, RS, Brasil.

${ }^{3}$ Programa de Pós-Graduação em Epidemiologia da Universidade Federal de Pelotas (UFPel). Pelotas, RS, Brasil.

${ }^{4}$ Departamento de Alimentos e Nutrição e Programa de Pós-Graduação em Gerontologia da Universidade Federal de Santa Maria (UFSM).

Santa Maria, RS, Brasil.<patriciachagas.ufsm@hotmail.com> (correspondente)
}

\begin{abstract}
INTRODUÇÃO: SARC-F+CP foi recentemente proposto como uma variação do questionário SARC-F, combinando suas questões (que avaliam a função muscular) com uma medida antropométrica [circunferência da panturrilha (CP), como substituto da massa muscular]. OBJETIVO: Avaliar o desempenho do SARC-F+CP como uma ferramenta de triagem de sarcopenia. MÉTODOS: Estudo transversal com idosas que realizaram densitometria óssea. A sarcopenia foi definida pelos critérios recomendados pelo European Working Group on Sarcopenia in Older People (EWGSOP): massa muscular avaliada através da absorciometria de duplo feixe de Raio-X (DXA); força muscular, avaliada pela força do aperto de mão; e rendimento físico, avaliado pelo teste de velocidade da marcha de 4 metros. Foram utilizados os valores específicos para a população brasileira para definir o ponto de corte do Índice de Massa Muscular Esquelética através da DXA $\left(5,62 \mathrm{~kg} / \mathrm{m}^{2}\right)$ e da $C P(\leq 33 \mathrm{~cm})$. A versão brasileira do SARC-F foi aplicada, e 10 pontos foram adicionados ao escore SARC-F se os sujeitos tivessem CP baixa. As idosas que apresentaram pontuação final $\geq 11$ foram consideradas com risco de sarcopenia. RESULTADOS: Um total de 205 idosas (idade média:67,3 $\pm 5,9$ anos) foram incluídas no estudo. A maioria da amostra era caucasiana (71,2\%), com 4-8 anos de estudo $(47,3 \%)$ e era aposentada (92,2\%). A prevalência de sarcopenia foi de 2,4\% da amostra total $(\mathrm{N}=5)$. Através do $\mathrm{SARCF}+\mathrm{CP}$, 37 mulheres (18\%) foram identificadas como apresentando risco de sarcopenia. Embora o SARC-F+CP tenha apresentado baixa sensibilidade $(40 \%)$ e valor preditivo positivo $(5,4 \%)$, ele apresentou bom desempenho na identificação de participantes saudáveis (especificidade: 82,5\%) e teve um excelente valor preditivo negativo (98,2\%). CONCLUSÃO: A combinação do SARC-F $+C P$, usando valores de corte regionais específicos para a $C P$, pode ser usada para excluir sujeitos saudáveis de novos testes, melhorando a triagem de sarcopenia na prática clínica.
\end{abstract}




\title{
Atendimento geriátrico gerontológico de enfermagem na atenção integral a saúde da pessoa idosa
}

\author{
Maria Helena Gehlen', Naiana Oliveira dos Santos², Claus Dieter Stobaus ${ }^{3}$ \\ Centro Universitário Franciscano e Instituto de Geriatria e Gerontologia da Pontifícia Universidade Católica do Rio Grande do Sul (IGG/PUCRS). \\ Porto Alegre, RS, Brasil.<gehlenmh@gmail.com> (correspondente) \\ ${ }^{2}$ Centro Universitário Franciscano. Santa Maria, RS, Brasil.<naiana.santos@unifra.br> \\ 3IGG/PUCRS. Porto Alegre, RS, Brasil.<stobaus@pucrs.br>
}

\begin{abstract}
INTRODUÇÃO: A promoção da atenção integral à saúde da pessoa idosa no atendimento geriátrico gerontológico, possui como foco a manutenção da sua funcionalidade global, que é a capacidade de gerir a própria vida ou cuidar de si mesmo, influenciada pelo seu grau de autonomia e independência. Objetivo: Descrever o atendimento geriátrico gerontológico de enfermagem, por meio da efetividade da consulta de enfermagem em uma Estratégia de Saúde da Família - ESF. MÉTODO: Estudo descritivo, do tipo relato de experiência, realizado por docentes e discentes de enfermagem, durante a consulta de enfermagem gerontológica em uma ESF, localizada na região central do Rio Grande do Sul, no período de fevereiro de 2015 a dezembro de 2017. Os idosos participantes foram os considerados robustos, com risco de fragilização e frágeis. RESULTADOS: Ao realizar o atendimento geriátrico gerontológico de enfermagem por meio da consulta de enfermagem gerontológica, foi possível avaliar, realizar o planejamento das intervenções e implementar continuamente os cuidados na manutenção das atividades básicas de vida diária, em garantia a longevidade com funcionalidade e promoção do bem-estar no cotidiano da pessoa idosa. CONCLUSÕES: $\bigcirc$ atendimento geriátrico gerontológico de enfermagem, desenvolvido na consulta de enfermagem promove a funcionalidade global da pessoa idosa considerando as suas dimensões educativas clinicas, espirituais, psicossociais, funcionais e socioambientais.
\end{abstract}




\title{
Obesidade e inatividade física em idosos do Brasil
}

\author{
Valéria Baccarin laniski', Renata Breda Martins², Flávia Picoli Gheno³ \\ 'Nutricionista, Especialista em Saúde da Família, aluna do curso de mestrado do Programa de Pós-Graduação em Gerontologia Biomédica, Escola de Medicina, \\ Pontifícia Universidade Católica do Rio Grande do Sul (PUCRS). Porto Alegre, RS, Brasil. < valeriaianiski@gmail.com> \\ ${ }^{2}$ Nutricionista, Especialista em Saúde do Idoso, aluna do curso de Mestrado do Programa de Pós-Graduação em Gerontologia Biomédica, PUCRS. \\ Porto Alegre, RS, Brasil. <nutri.renatamartins@gmail.com> \\ ${ }^{3}$ Nutricionista, Mestre em Gerontologia Biomédica, PUCRS. Porto Alegre, RS, Brasil. \\ <flavia.brenda@gmail.com> (correspondente)
}

\begin{abstract}
INTRODUÇÃO: As mudanças fisiológicas e estruturais do envelhecimento corroboram para o acontecimento de alterações no perfil nutricional e redução da mobilidade nos idosos. OBJETIVO: Descrever a proporção de obesidade e idosos fisicamente inativos no Brasil. METODOLOGIA: Estudo transversal, com dados SISAP-idoso. A obesidade (IMC $\left.>30 \mathrm{~kg} / \mathrm{m}^{2}\right)$ refere-se à Pesquisa Nacional de Saúde (PNS, 2013) e dados de idosos fisicamente inativos são provenientes da Pesquisa Nacional por Amostra de Domicílios (2008) e PNS. RESULTADOS: No Brasil a proporção de idosos obesos foi de 23,3\%, sendo maior nas mulheres $(27,8 \%)$. Nas regiões, a região Sul $(28,9 \%)$ apresentou maior proporção de obesidade, assim como, também foi observada em relação aos gêneros nesta região (mulheres: 31,85\%, homens: 25,0\%). Santa Catarina foi o estado com maior proporção de obesidade $(33,5 \%)$ iá, nas mulheres maiores proporções foram observadas no Mato Grosso $(37,2 \%)$ e em homens, no Rio Grande do Sul (25,7\%). A proporção de idosos fisicamente inativos no país passou de 33,4\% (2008) para 7,1\% (2013), sendo maior nos homens (2008:40,3\%; 2013:10,6\%). Em 2008 a proporção de idosos inativos era maior no Norte (39,10\%), e em 2013, foi maior no Centro-Oeste $(8,4 \%)$. Homens apresentaram maior proporção de inatividade física nos diferentes anos (2008: 44,5\%; 2013: 12,0\%). As maiores proporções de idosos inativos foram observadas no Pará $(43,0 \%, 2008)$ e no Amapá (11,6\%, 2013). Houve maior proporção de homens inativos no Amazonas $(48,6 \%, 2008)$ e no Rio Grande do Sul (16,82\%, 2013). CONCLUSÕES: As mulheres apresentaram maior proporção de obesidade nas regiões Sul e Centro-Oeste. Já, os homens mostraram-se mais fisicamente inativos no Norte e Centro-Oeste e em seus estados, com exceção em 2013. Os achados sugerem que a obesidade é influenciada por outros fatores de estilo de vida além da inatividade física.
\end{abstract}




\section{Variáveis antropométricas e sua associação com a velocidade da marcha em idosas socialmente ativas}

\section{Karen Mello de Mattos Margutti' , Adriane Rosa Costodio², Maria Luiza Freitas Annes ${ }^{3}$, Tereza Cristina Blasi ${ }^{4}$ Natielen Jacques Schuch ${ }^{5}$; Carla Helena Augustin Schwanke ${ }^{6}$}

'Nutricionista. Mestre em Saúde Coletiva. Doutora em Gerontologia Biomédica, Escola de Medicina da Pontifícia Universidade Católica do Rio Grande do Sul (PUCRS).Porto Alegre, RS, Brasil.<kmmattos@yahoo.com.br>

${ }_{2}$ Nutricionista. Mestranda do Programa de Pós-Graduação em Gerontologia da Universidade Federal de Santa Maria (UFSM). Santa Maria, RS, Brasil. <adricostodiosm@yahoo.com.br>

${ }^{3}$ Nutricionista, aluna do curso de Doutorado do Programa de Pós-Graduação em Gerontologia Biomédica (GERONBIO), PUCRS. Bolsista CAPES.

<maluannes@hotmail.com> (correspondente)

${ }^{4}$ Nutricionista. Mestre em Tecnologia dos Alimentos. Professora do curso de Nutrição do Centro Universitário Franciscano. Santa Maria, RS, Brasil. <tcb29@hotmail.com>

${ }^{5}$ Nutricionista. Doutora em Nutrição em Saúde Pública. Professora do curso de Nutrição e do Programa de Mestrado em Ciências da Saúde e da Vida do Centro Universitário Franciscano. Santa Maria, RS, Brasil. <natielen@yahoo.com.br>

${ }^{6}$ Geriatra. Doutora em Gerontologia Biomédica. Professora permanente do Programa de Pós-Graduação em Gerontologia Biomédica do Instituto de Geriatria e Gerontologia da Pontifícia Universidade Católica do Rio Grande do Sul (IGG/PUCRS). Porto Alegre, RS, Brasil.

<schwanke@pucrs.br>

INTRODUÇÃO: A velocidade da marcha consiste em um bom indicador para avaliar o declínio funcional e mobilidade física do idoso e pode ser alterada por modificações no sistema musculoesquelético e composição corporal decorrentes do envelhecimento. OBJETIVO: Avaliar a associação entre as variáveis antropométricas e a velocidade da marcha em idosas socialmente ativas. MÉTODOS: Estudo transversal realizado com idosas da comunidade socialmente ativas, recrutadas em grupos de convivência de Santa Maria/RS. Foram analisadas as variáveis: idade (anos), peso $(\mathrm{kg})$, estatura $(\mathrm{m})$, Índice de Massa Corporal - IMC $\left(\mathrm{kg} / \mathrm{m}^{2}\right)$, circunferência da cintura $(\mathrm{cm})$, razão cintura-estatura (RCE) e velocidade da marcha. A velocidade da marcha foi avaliada em um percurso de quatro metros e classificada inadequada quando $<0,8$ metros/segundo $(\mathrm{m} / \mathrm{s})$. Para a análise estatística foi utilizado o programa SPSS Statistics ${ }^{\circledR}$ versão 22.0, sendo significativo o valor de $P<0,05$. RESULTADOS: Participaram do estudo 344 idosas com média de idade de 69,95 $\pm 6,28$ anos (faixa de 60-89 anos) e de velocidade da marcha de 0,87 $\pm 0,20 \mathrm{~m} / \mathrm{s}$. Idosas com velocidade da marcha inadequada apresentaram maiores médias de peso $[69,64 \pm 13,63 \mathrm{~kg} \times 65,89 \pm 11,13 \mathrm{~kg}(P=0,008)]$, IMC $\left[29,09 \pm 5,24 \mathrm{~kg} / \mathrm{m}^{2} \times 27,05 \pm 4,19 \mathrm{~kg} / \mathrm{m}^{2} \quad(P=0,001)\right]$, circunferência da cintura $[98,97 \pm 11,36 \mathrm{~cm} \times 94,01 \pm 9,99$ $(P<0,001)], \operatorname{RCE}[0,64 \pm 0,07 \times 0,60 \pm 0,06(P<0,001)]$ e menores médias de estatura $[1,54 \pm 0,06 \mathrm{~m} \times 1,55 \pm 0,06 \mathrm{~m}(P=0,035)]$ quando comparadas com idosas com a velocidade da marcha adequada. CONCLUSÕES: A inadequação da velocidade da marcha mostrou-se associada com maior peso, IMC, circunferência da cintura e RCE. Resultados que reforçam a relação entre a maior adiposidade e excesso de peso com uma menor capacidade funcional em idosos. 


\title{
Função pulmonar, força muscular inspiratória e sintomas cardiorrespiratórios em nonagenários: estudo AMPAL
}

\author{
Josemara de Paula Rocha', Fabiane de Oliveira Brauner², Gabriela Guimarães Oliveira3 \\ Bruna Rios Rauber ${ }^{4}$, Marlon Cássio Pereira Grigol ${ }^{5}$, Ângelo José Gonçalves Bós ${ }^{6}$ \\ 'Instituto de Geriatria e Gerontologia da Pontifícia Universidade Católica do Rio Grande do Sul (IGG/PUCRS). Porto Alegre, RS, Brasil. \\ <josemara.rocha@hotmail.com> (correspondente) \\ 2IGG/PUCRS. Porto Alegre, RS, Brasil. <braunerpro@gmail.com> \\ 3IGG/PUCRS. Porto Alegre, RS, Brasil.<oliveira gabriela@hotmail.com> \\ 4IGG/PUCRS. Porto Alegre, RS, Brasil.<brurauber@gmail.com> \\ 5IGG/PUCRS. Porto Alegre, RS, Brasil.<marlonfisio@gmail.com> \\ ${ }^{6}$ GGG/PUCRS. Porto Alegre, RS, Brasil.<angelo.bos@pucrs.br>
}

\begin{abstract}
INTRODUÇÃO: As doenças respiratórias estão entre as principais causas de morte na longevidade. A função pulmonar (FP) e a força inspiratória (FI) são importantes fatores de risco para essas doenças, com poucos estudos em nonagenários e seus sintomas. OBJETIVOS: Investigar a FP e a FI em nonagenários e sua relação com doença e sintomas cardiorrespiratórios. MÉTODOS: Estudo transversal analítico em nonagenários comunitários, não institucionalizados, do estudo Atenção Multiprofissional ao Longevo - AMPAL da Pontifícia Universidade Católica do Rio Grande do Sul, representativos de Porto Alegre/RS. A FP foi avaliada por microespirômetro portátil Carefusion ${ }^{\text {TM }}$ mensurando: volume expiratório forçado no primeiro segundo $\left(V E F_{1}\right)$, capacidade vital forçada (CVF) e relação $V E F_{1} / C V F$ com valores preditos (pred) pela American Thoracic Society/ European Respiratory Society. A FI foi avaliada pela manovacuometria digital (MVD300 ${ }^{\mathrm{TM}}$ ) através da pressão inspiratória máxima (Plmáx). Investigou-se o diagnóstico de pneumopatia e sintomas cardiorrespiratórios nos últimos dois meses: fadiga, falta de ar e sibilo, entre outros. A dispneia foi classificada pela escala Medical Research Council (MRC) de 1 a 5 pontos (5 o mais grave). Médias comparadas pela análise de variância, aceitando $p<0,05$ como significativo. RESULTADOS: Participaram 64 nonagenários, $67 \%$ mulheres, 93 2 2, 8anos, $14 \%$ pneumopatas, 33\% com fadiga, 27\% com falta de ar, $6 \%$ com sibilo, $36 \%$ tinha MRC grau 1, valores absolutos de FP: VEF $1,6 \pm 0,6 \mathrm{~L} / \mathrm{s}, \mathrm{CVF} 2,06 \pm 0,73 \mathrm{~L}, \mathrm{VEF}_{1} / \mathrm{CVF} 78,3 \pm 11,3 \%$, valores preditos: $\mathrm{VEF}_{1 \text { pred }} 118,6 \pm 34,7 \%, \mathrm{CVF}_{\text {pred }} 114,9 \pm 31,1 \%, \mathrm{VEF}_{1} / \mathrm{CVF}_{\text {pred }} 110,1 \pm 15,8 \%$, Plmáx $-45,5 \pm 16,0 \mathrm{cmH}_{2} \mathrm{O}$. CONCLUSÕES: Os valores de FP encontrados em nonagenários foram próximos aos poucos estudos internacionais com essa população etária, já os de PImáx foram de difícil comparação. A FP se relacionou com pneumopatia e tanto a FP quanto a Plmáx apresentaram relação significativa com fadiga, falta de ar e a escala de dispneia da MRC.
\end{abstract}




\title{
Caracterização dos estomizados atendidos na atenção primária em município do Rio Grande do Sul
}

\author{
Valéria Baccarin laniski', Renata Breda Martins², Letícia Mazocco33, Eniva Miladi Fernandes Stumm ${ }^{4}$, \\ Ana Karina Silva da Rocha Tanaka ${ }^{5}$, Claudine Lamanna Schirmer ${ }^{6}$, Carla Helena Augustin Schwanke ${ }^{7}$ \\ 'Nutricionista, egressa do Programa de Residência Multiprofissional em Saúde da Família UNIJUÍ/FUMSSAR. Mestranda do Programa de Pós-Graduação \\ em Gerontologia Biomédica (GERONBIO), Escola de Medicina, Pontifícia Universidade Católica do Rio Grande do Sul (PUCRS). \\ Bolsista CAPES/PROEX. Porto Alegre, RS. Brasil. \\ ${ }^{2}$ Nutricionista. Mestranda do Programa de Pós-Graduação em Gerontologia Biomédica, PUCRS. Bolsista CNPq. Porto Alegre, RS. Brasil. \\ 3Nutricionista. Doutoranda do Programa de Pós-Graduação em Gerontologia Biomédica, PUCRS. Bolsista CAPES/PROEX. Porto Alegre, RS. Brasil. \\ ${ }^{4}$ Enfermeira. Doutora em Ciências-Enfermagem pela Universidade Federal de São Paulo. Professora do Departamento de Ciências da Vida (DcVida) da \\ Universidade Regional do Noroeste do Estado do Rio Grande do Sul (UNIJUÍ). Porto Alegre, RS. Brasil. \\ ${ }^{5}$ Enfermeira, graduada pela UNISINOS. Mestre e Doutora em Gerontologia Biomédica, EM/PUCRS. Docente da Escola de Enfermagem da \\ Universidade Federal do Rio Grande do Sul (UFRGS). Porto Alegre, RS. Brasil. \\ ${ }^{6}$ Nutricionista. Especialista em Saúde da Família e Comunidade. Mestre e doutoranda em Gerontologia Biomédica, PUCRS. Porto Alegre, RS. Brasil. \\ Geriatra. Doutora em Gerontologia Biomédica. Professora do Programa de Pós-Graduação em Gerontologia Biomédica, PUCRS. Porto Alegre, RS. Brasil.
}

\begin{abstract}
INTRODUÇÃO: A estomização decorre de um procedimento cirúrgico que acomete indivíduos de diferentes faixas etárias e relaciona-se com neoplasias intestinais bem como, com a agudização de doenças crônicas. OBJETIVO: Caracterizar indivíduos estomizados permanentes que são atendidos pela atenção primária em saúde em município localizado no noroeste do Rio Grande do Sul, Brasil. MÉTODOS: Estudo transversal, com dados secundários obtidos do registro do sistema de Gerenciamento de Usuários com Deficiência da Atenção Primária no ano de 2017. As variáveis do estudo foram: sexo, faixa etária (20-59 anos: adultos; $\geq 60$ anos: idosos), área de moradia (rural e urbano), tipos de estomia (colostomia; ileostomia e urostomia), doença de base para estomização e tempo de cirurgia (meses). As doenças de base foram classificadas de acordo com o Código Internacional de Doenças (CID-10). Os dados foram analisados por meio de frequência simples, média e desvio padrão, mediana e intervalo interquartil, com auxílio do software estatístico SPSS 20.0. RESULTADOS: Participaram do estudo 69 indivíduos estomizados com média de idade de 60,62 16,74 anos (23 a 96 anos), sendo 58\% ( $n=40$ ) homens e $42 \%$ ( $n=29)$ mulheres. Os indivíduos em sua maioria eram idosos $(60 \%, n=41)$, moradores de área urbana $(81 \%, n=56)$ e apresentavam colostomia $(86 \%, n=59)$. As doenças de base mais frequentes foram neoplasia maligna do reto $(21,74 \%, n=15)$, neoplasia maligna do cólon $(20,3 \%, n=14)$ e doença diverticular do intestino $(14,5 \%, n=10)$, com mediana de tempo de 24 meses $(8,5-54$ meses). CONCLUSÃO: Observou-se uma predominância de idosos, homens, moradores da área urbana, com colostomia há 24 meses em decorrência de afecções intestinais.
\end{abstract}




\title{
Conhecimento de estudantes de medicina sobre medicamentos inapropriados para idosos: estudo em alunos dos últimos semestres do Curso de Medicina ULBRA-Canoas
}

\author{
Alessandra S. Menin', Crissiane M. Nepomuceno ${ }^{2}$, Isadora B. da Silva², Paulo R. C. Consoni² \\ 'Universidade Luterana do Brasil (ULBRA). Canoas, RS, Brasil. <asmenin@icloud.com> (correspondente) \\ ${ }^{2}$ ULBRA. Canoas, RS, Brasil.
}

\begin{abstract}
INTRODUÇÃO: Reações adversas a medicamentos são frequentes e mais graves em idosos. A polifarmácia e a prescrição de medicamentos inapropriados expõem esses pacientes a riscos, aumentando a morbimortalidade. A falta de conhecimento e treinamento inadequado dos profissionais da saúde em relação à prescrição para o paciente idoso contribuem para a iatrogenia medicamentosa. OBJETIVO: Descrever o conhecimento de alunos de medicina sobre medicamentos inapropriados para idosos. MÉTODO: Estudo transversal com questionários aplicados a 102 alunos dos 137, matriculados do 90 ao 11 o semestre da Medicina da ULBRA-Canoas. Levantaram-se informações referentes ao conhecimento dos acadêmicos sobre medicamentos inapropriados para idosos, questionando sobre a segurança da prescrição de certos fármacos de uso rotineiro na rede pública de saúde, para um paciente idoso frágil ou com mais de 80 anos, baseado na classificação do Critério de Beers. Análise estatística feita com o SPSS 21.0, e testes Qui-Quadrado para comparar as variáveis. RESULTADOS: 84,3\% dos alunos pesquisados afirmaram ter conhecimento sobre medicamentos inapropriados para idosos, mas o percentual de acertos nos questionários foi de 20,2\%. O maior número de acertos foi quanto à segurança do Diazepam (49\%). Já o menor percentual foi com o fármaco Fluoxetina, com 4,9\% das respostas corretas. Os fármacos com maior percentual de respostas "Não Sei" foram Oxibutinina (48\%) e Ciclobenzaprina (37,3\%). CONCLUSÃO: Mesmo que a maioria dos alunos afirmara conhecer o tema, a frequência de acertos quanto à segurança dos fármacos questionados foi baixa. O aprimoramento do ensino na graduação faz-se necessário para minimizar a iatrogenia em pacientes idosos.
\end{abstract}




\title{
Avaliação da funcionalidade de idosos institucionalizados: estudo transversal
}

\author{
Crissiane M. Nepomuceno', Alessandra S. Menin², Victória S. W. Soriano², Paulo R. C. Consoni² \\ Universidade Luterana do Brasil (ULBRA). Canoas, RS, Brasil. <crismelonepo@hotmail.com> (correspondente) \\ 2 ULBRA. Canoas, RS, Brasil.
}

INTRODUÇÃO: O crescimento da população idosa no Brasil exige mudanças nas políticas públicas. Assim, muitos indivíduos de ILPls apresentam alguma vulnerabilidade e dependem da ajuda de cuidadores para realizar suas atividades diárias. OBJETIVO: Avaliar a capacidade funcional do indivíduo idoso institucionalizado. MÉTODO: Realizou-se o estudo numa ILPI municipal em Canoas, em março de 2017. Todos os residentes com idade igual ou superior a 60 anos participaram da coleta de dados. Utilizou-se a Escala de Katz para análise do grau de funcionalidade. RESULTADOS: A população da ILPI era de 45 idosos, dois foram excluídos do estudo, um pela idade inferior a 60 anos e outro pela ausência. Logo, a amostra do estudo foi de 43 idosos, 55,8\% sexo feminino e 44,1\% masculino. A idade média foi 76,7. No item banho, 51,1\% não recebe assistência, 11,6\% recebe para uma parte do corpo e 37,2\% não toma banho sozinho. No vestuário, 48,8\% afirmou vestir-se sem assistência, $11,6 \%$ recebe assistência para amarrar os sapatos e 39,5\% veste-se com assistência. Na avaliação de higiene pessoal, 60,4\% vai ao banheiro sem assistência, 39,5\% recebe assistência. No item transferência, 58,1\% deita, levanta e senta sem assistência, 41,8\% deita, levanta e senta com assistência. Para continência, 48,8\% apresenta controle esfincteriano completo, $11,6 \%$ com acidentes ocasionais e 39,5\% com supervisão, uso de cateter ou incontinente. Na alimentação, $90,7 \%$ se alimenta sem assistência, 4,6\% com assistência para cortar carne/manteiga no pão e 4,5\% com assistência, sondas ou fluidos intravenosos. CONCLUSÃO: Já que 70\% dos idosos apresentou algum grau de dependência, conclui-se o quão importante é a multiprofissionalidade em ILPIs a fim de que todos os idosos dependentes sejam atendidos. 


\title{
Capacidade funcional da pessoa idosa: utilização da Escala de Barthel na avaliação das atividades básicas de vida diária
}

\author{
Lucimara Sonaglio Rocha', Marlene Teda Pelzer ${ }^{2}$, Andressa Peripolli Rodrigues ${ }^{3}$, \\ Neiva Claudete Brondani Machado ${ }^{4}$, Margot Agathe Seiffert ${ }^{5}$, Elizabete Marta Krebs 6 , \\ Rita Fernanda Monteiro Fernandes 7 , Sandra Maria de Mello Cardoso ${ }^{8}$
}

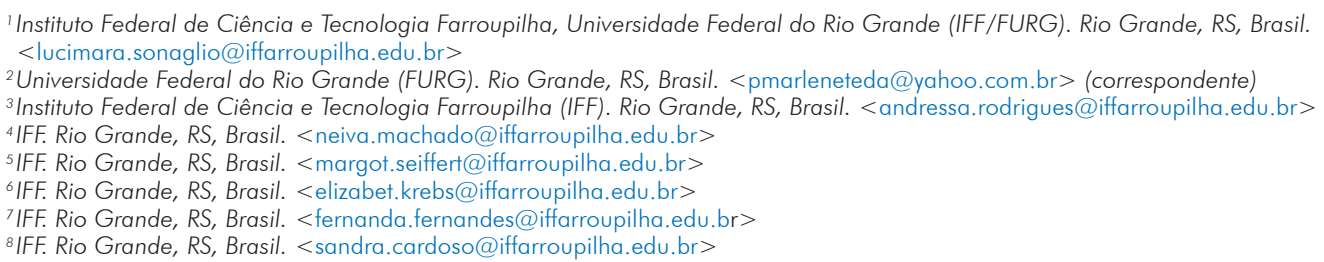

INTRODUÇÃO: A avaliação funcional é essencial para o estabelecimento de um diagnóstico, um prognóstico e um julgamento clínico adequados os quais servirão de base para as decisões sobre os tratamentos e cuidados necessários à manutenção da independência e autonomia da pessoa idosa. Nesse sentido, o desempenho das atividades básicas de vida diária (ABVD) é considerado um parâmetro aceito e legítimo para firmar essa avaliação. OBJETIVO: $\bigcirc$ presente trabalho tem por objetivo apresentar uma das escalas para a avaliação das atividades básicas de vida diária: a escala de Barthel. METODOLOGIA: Trata-se de uma revisão da literatura baseada em estudos especializados disponíveis em artigos científicos e obras de referência da área de geriatria e gerontologia. RESULTADOS: A Escala de Barthel, foi formulada originalmente por Mahoney e Barthel (1965) para avaliação de pacientes vítimas de acidente vascular cerebral, mas mostrou-se eficiente na avaliação de pessoas idosas em geral. Na Escala também denominada Índice ou Index de Barthel, cada item é pontuado de acordo com o desempenho do paciente em realizar tarefas de forma independente, com alguma ajuda ou de forma dependente. Esta escala avalia dez funções: tomar banho, vestir-se, promover higiene, usar o vaso sanitário, transferir-se da cama para cadeira e vice-versa, manter continências fecal e urinária, capacidade para alimentar-se, deambular e subir e descer escadas. A escala de Barthel permite uma gradação mais ampla na classificação da dependência quando comparada com a Escala de Katz, indo desde a dependência total (0 ponto) a independência máxima (100 pontos). O instrumento foi submetido em 2007 ao processo de adaptação transcultural e fora demonstrada sua confiabilidade interexaminadores $(0,95)$ e confiabilidade teste-resteste $(0,89)$, assim como fortes correlações $(0,74$ a 0,80) com outras medidas de incapacidade. CONCLUSÕES: A Escala de Barthel é um dos instrumentos para a avaliação das ABVD mais utilizados em pessoas idosas a nível internacional dada a facilidade de sua aplicação e interpretação, avaliação do item deambulação e forte confiabilidade e correlação com outras medidas de incapacidade. 


\section{Revisão de literatura: inclusão digital para idosos}

\section{Thayze Maria Marques Torbes', Bruna Camargo², Juliana Mello Severo ${ }^{3}$, Cecília Dias Flores 4 , Tamara Rosa Lansini Pereira Tolotti ${ }^{5}$, Mariana Edinger Wieczorek ${ }^{6}$, Mariane de Moura Fernandes ${ }^{7}$, lohana Schroder Bubolz ${ }^{8}$}

'Universidade Federal de Ciências da Saúde de Porto Alegre (UFCSPA). Porto Alegre, RS, Brasil. <thayzetorbes@hotmail.com>

2UFCSPA. Porto Alegre, RS, Brasil. < bcamargo@ufcspa.edu.br>

3UFCSPA. Porto Alegre, RS, Brasil.<julianase@ufcspa.edu.br>

${ }^{4}$ UFCSPA. Porto Alegre, RS, Brasil. <dfloresorama@gmail.com>

5UFCSPA. Porto Alegre, RS, Brasil. <tamara.lansini@gmail.com>

○UFCSPA. Porto Alegre, RS, Brasil. <mariana.ew@hotmail.com>

7UFCSPA.Porto Alegre, RS, Brasil.<maridemourafernandes@gmail.com>

¿UFCSPA. Porto Alegre, RS, Brasil. <iohanasb@hotmail.com>

INTRODUÇÃO: $\bigcirc$ envelhecimento humano é um processo natural e gradual que ocorre de maneira individual. Tendo em vista que a população idosa tem mostrado grande crescimento e tende a aumentar nas próximas décadas e que a tecnologia se faz cada vez mais presente no cotidiano, torna-se relevante estudar a relação entre os idosos e o meio digital. OBJETIVOS: Revisar na literatura as teses e dissertações elaboradas com a temática de inclusão digital na população idosa. MÉTODOS: Trata-se de uma revisão de literatura realizada no Catálogo de Teses e Dissertações da CAPES, sendo utilizados os termos inclusão digital, informática e idosos. Utilizou-se o operador lógico booleano "AND" para restringir a pesquisa, sendo feitas duas buscas com as seguintes estratégias: inclusão digital AND idosos, informática AND idosos. Foram incluídos neste estudo trabalhos que abordassem a inclusão de idosos no meio digital, não sendo restringido à área de ciências médicas, mas incluindo diversas áreas do conhecimento. RESULTADOS: Dentre os 47 trabalhos encontrados entre 1999 e 2016, mais da metade (27) foi produzida a partir de 2011 , demonstrando acréscimo recente em pesquisas na área. Ainda, a maior parte dos estudos tem como temática "Computação e áreas afins", "Educação, Comunicação, Cognição e Linguagem" ou "Gerontologia e Envelhecimento Humano". Por outro lado, áreas como "Psicologia", "Desenvolvimento Humano" e "Sociologia" totalizam juntas apenas aproximadamente 10\% das produções. CONCLUSÕES: Há poucos estudos que analisam a inclusão digital para os idosos. São necessários mais estudos com essa temática para que se tenha mais dados relacionados aos fatores que interferem na interação do idoso com o meio digital. 


\section{Condições e modo de vida das pessoas idosas em situação de rua}

\section{Carine Magalhães Zanchi de Mattos' ${ }^{1}$ Tamara Rosa Lansini Pereira Tolotti ${ }^{2}$, Bruna Camargo ${ }^{3}$, Patricia Krieger Grossi ${ }^{4}$, Mariana Petraco Miranda ${ }^{5}$, Giovana Petraco Miranda ${ }^{6}$}

'Pontifícia Universidade Católica do Rio Grande do Sul (PUCRS).Porto Alegre, RS, Brasil. < carinezanchi@gmail.com>

2Universidade Federal de Ciências da Saúde de Porto Alegre (UFCSPA). Porto Alegre, RS, Brasil. <tamara.lansini@gmail.com>

3UFCSPA. Porto Alegre, RS, Brasil. < bcamargo@ufcspa.edu.br>

${ }_{4}^{4}$ PUCRS. Porto Alegre, RS, Brasil. <pkgrossi@pucrs.br>

PUCRS. Porto Alegre, RS, Brasil.<marianapetracco@gmail.com>

bPUCRS. Porto Alegre, RS, Brasil. <gipetracco@gmail.com>

INTRODUÇÃO: As condições e o modo de vida de idosos em situação de rua requerem atenção devido à complexidade de fenômenos que os envolve como transição demográfica na rua, dificuldade de reinserção social, desemprego estrutural, desconstrução social e desvalorização do indivíduo. Essa camada persiste na miséria extrema, desvinculada da produção social e se dedicando a atividades marginais. OBJETIVOS: Compreender as condições e o modo de vida para pessoas com mais de 60 anos de idade que vivem em situação de rua em Porto Alegre. MÉTODOS: Estudo descritivo de abordagem qualitativa no qual foram selecionados 19 idosos em situação de rua, por amostragem aleatória simples e fechamento amostral por saturação teórica, entrevistados no período de 2015 a 2017 . Foi realizada análise textual discursiva. RESULTADOS: Os pesquisados têm entre 60 e 69 anos, são negros ou pardos e têm ensino fundamental incompleto. Têm como estratégias de sobrevivência o acesso a locais movimentados e centros de acolhimento institucional, vínculos com conhecidos que doam roupas e alimentos, trabalho informal e, no caso das mulheres, o relacionamento com um companheiro. Suas experiências sociais são influenciadas por marcadores de diferença de gênero, etnia, território, classe social e condição física. Sofrem violência estrutural e interseccional, vivenciadas desde o nascimento. Para as pessoas em situação de rua, qualidade de vida seria ter outra história, com acesso a saúde, alimentação, família/cuidador/esposa e um lugar para morar. CONCLUSÕES: Observou-se a importância de criar espaços para discutir o tema a nível nacional, acadêmico e no âmbito das políticas públicas, dando voz para que essa população expresse suas demandas e para que o Estado possa garantir os direitos básicos. 


\title{
Avaliação nutricional versus funcionalidade em idosos residentes em Instituição de Longa Permanência em cidade metropolitana do Rio Grande do Sul, no período de abril e junho de 2018
}

\author{
Alessandra S. Menin', Crissiane M. Nepomuceno', Paulo R. C. Consoni', Raul U. Hilbig', \\ Aline da C. Gobbi' ${ }^{2}$ Caroline G. Covatti', Michele B. Closs' ${ }^{1}$, Miria E. B. de Camargo' \\ Universidade Luterana do Brasil (ULBRA). Canoas, RS, Brasil. \\ 2ULBRA. Canoas, RS, Brasil.<alinec.gobbi@gmail.com> (correspondente)
}

\begin{abstract}
INTRODUÇÃO: No processo de envelhecimento, é relevante o tema da nutrição geriátrica, visto o impacto na saúde e qualidade de vida de idosos. Aliando-se o tema da funcionalidade com nutrição, tem-se apontado o estado nutricional como um importante fator de proteção de perda da funcionalidade do idoso da comunidade. OBJETIVO: Conhecer a relação entre o estado nutricional e a funcionalidade em idosos de ambos os gêneros residentes de uma ILPI no RS. METODOLOGIA: Realizou-se um estudo transversal de avaliação direta e análise de prontuário, utilizando o teste de Mini Avaliação Nutricional (MAN) e o Índice de Katz. Para o processamento de dados e controle de qualidade foram empregados dois Softwares: SPSS e MINITAB, além de revisão de literatura. Houve aprovação do estudo pelo Comitê de Ética e Pesquisa da ULBRA. RESULTADOS: Foram avaliados 40 idosos com idade média de 70 anos. Em relação ao estado nutricional, 37,5\% tem avaliação nutricional normal, $50 \%$ estão em risco de má nutrição e 12,5\% em má nutrição. Em relação à funcionalidade, $80 \%$ encontram-se independentes, $9 \%$ parcialmente dependentes e $11 \%$ com dependência total. Segundo a análise estatística, há uma relação significativa entre as duas variáveis (Avaliação Nutricional x Funcionalidade), sendo a correlação de Sepaman's = 0,47 - valor maior que 0,1 ou seja, considerado positivo. CONCLUSÃO: Observou-se que existe uma relação estatisticamente significativa entre o estado nutricional e a capacidade funcional, sendo que quanto melhor for o estado nutricional de idosos residentes na instituição de longa permanência, menor é o grau de dependência.
\end{abstract}




\title{
Sarcopenia em idosos com doença renal crônica: uma revisão integrativa
}

\author{
Luana Fioravanti Roland', Vítor Buss', Carla Cristina Bauermann Brasil2, Loiva Beatriz Dallepiane \\ 'Nutricionista; Mestranda(o) do Programa de Pós-Graduação em Gerontologia, Universidade Federal de Santa Maria (UFSM). Santa Maria, RS, Brasil. \\ ${ }_{2}^{2}$ Professora Doutora do Departamento de Alimentos e Nutrição, Curso de Nutrição, UFSM. Santa Maria, RS, Brasil. \\ ${ }^{3}$ Professora Doutora do Departamento de Alimentos e Nutrição, Curso de Nutrição e do Programa de Pós-Graduação em Gerontologia, UFSM. \\ Santa Maria,RS, Brasil.<luanafioravanti.ufsm@gmail.com>
}

\begin{abstract}
INTRODUÇÃO: A sarcopenia é uma condição associada ao envelhecimento patológico e é definida pela redução de massa muscular e força e/ou desempenho muscular, impactando negativamente na qualidade de vida do idoso. Na Doença Renal Crônica (DRC) a sarcopenia tem elevada prevalência e está associada com múltiplos distúrbios metabólicos e nutricionais, bem como com o aumento da morbimortalidade. OBJETIVO: Buscar na literatura científica estudos relacionados à sarcopenia em idosos com doença renal crônica. METODOLOGIA: Revisão bibliográfica de artigos publicados nos últimos 5 anos e indexados na base de dados PUBMED, com desenho de estudos transversais sobre sarcopenia em idosos com DRC, sendo que a busca ocorreu com os descritores "sarcopenia", "aging" e "chronic renal disease". RESULTADOS: Foram encontrados 10 artigos, dos quais 3 foram excluídos por não abordarem a temática da pesquisa. Na análise do restante, observou-se que a sarcopenia está associada com marcadores inflamatórios, $\beta-2$ microglobulina, depressão e declínio cognitivo. Ainda a perda proteico-calórica está interligada à inflamação e mortalidade em idosos sarcopênicos com DRC em fase terminal. Outro fator que vale destacar é que há associação da sarcopenia com o declínio da filtração glomerular. Como limitação do estudo destaca-se o tamanho amostral e o curto período de busca. CONCLUSÕES: Os idosos com DRC devem ser submetidos a medidas preventivas e avaliados quanto à presença de sarcopenia em estágios mais precoces. Entretanto ainda há uma lacuna no que diz respeito a esse assunto, e mais trabalhos são necessários para uma maior compreensão da fisiopatologia, implicações clínicas, diagnóstico e abordagem terapêutica.
\end{abstract}




\title{
Intervenção fonoaudiológica precoce para afasia transcortical motora - estudo de caso
}

\author{
Helena C. B. Rosa', Andressa O. Birck², Mariane de M. Fernandes ${ }^{3}$, Luiza M. T. V. Lima ${ }^{4}$, \\ Fernanda Loureiro ${ }^{5}$, Bárbara C. Beber ${ }^{6}$
}

\author{
Departamento de Fonoaudiologia, Universidade Federal de Ciências da Saúde de Porto Alegre (UFCSPA). Porto Alegre, RS, Brasil. \\ <helena.rosa91@yahoo.com.br> \\ 2Departamento de Fonoaudiologia, UFCSPA.Porto Alegre, RS, Brasil.<andressabirck@hotmail.com> \\ 3Departamento de Fonoaudiologia, UFCSPA. Porto Alegre, RS, Brasil. <maridemourafernandes@gmail.com> \\ ${ }^{4}$ Departamento de Fonoaudiologia, UFCSPA. Porto Alegre, RS, Brasil.<lumtvlima@gmail.com> \\ ${ }^{5}$ Programa de Pós-Gradução em Gerontologia Biomédica, PUCRS. Porto Alegre, RS, Brasil. <fernanda.loureiro@pucrs.br> \\ 6Departamento de Fonoaudiologia, UFCSPA. Porto Alegre, RS, Brasil. <barbaracb@ufcspa.edu.br>
}

\begin{abstract}
INTRODUÇÃO: A afasia transcortical motora (ATM) é um transtorno de linguagem não fluente causada por danos em áreas motoras do lobo frontal esquerdo. Durante os primeiros meses pós-lesão há uma recuperação espontânea, e a intervenção fonoaudiológica neste período pode potencializar a recuperação do paciente. OBJETIVO: Verificar o efeito da intervenção fonoaudiológica precoce em um caso de ATM. MÉTODOS: Paciente do sexo feminino, 45 anos de idade, 8 anos de escolaridade, diagnosticada com ATM em decorrência de acidente vascular encefálico (AVE). As sessões de fonoterapia foram realizadas em um hospital filantrópico da cidade de Porto Alegre um mês após o AVE, com duas estagiárias de fonoaudiologia e a supervisão de duas fonoaudiólogas, totalizando 10 sessões semanais de 40 minutos cada, além de exercícios para realizar em casa. Utilizou-se a bateria Montreal Tolouse de Avaliação da Linguagem (MTL-BRASIL) para a avaliação pré e após 10 sessões de fonoterapia. RESULTADOS: A paciente apresentou melhora de três pontos ou mais no desempenho das tarefas de compreensão oral, repetição e praxias não-verbais; e um ou dois pontos nas tarefas de linguagem automática, compreensão escrita, nomeação oral e reconhecimento de partes do corpo e noções de direita e esquerda. CONCLUSÃO: Houve melhora no desempenho de diversas habilidades linguísticas, devido não só ao período de recuperação espontânea, mas também à intervenção fonoaudiológica precoce.
\end{abstract}




\title{
Análise do perfil de pacientes idosos do setor de fonoaudiologia de uma UBS de Porto Alegre
}

\author{
Luíza Menegaz T. V. Lima', Mariane de Moura Fernandes², Andressa Birck³, Helena Cristina B. Rosa ${ }^{4}$, \\ Andrea W. Bonamigo ${ }^{5}$, Fabiana de Oliveira ${ }^{6}$, Bárbara C. Beber ${ }^{7}$
}

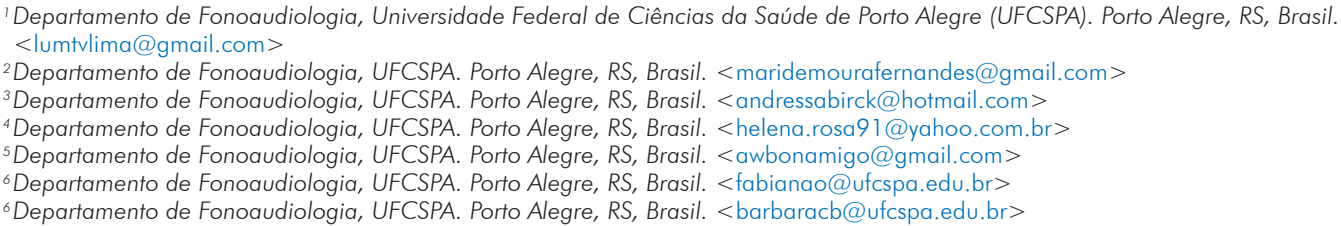

INTRODUÇÃO: $\bigcirc$ envelhecimento populacional é um desafio para sistemas de saúde e de previdência social em todo o mundo. É de extrema importância que os serviços de saúde conheçam o perfil epidemiológico de suas populações para um melhor planejamento da assistência. OBJETIVO: Descrever o perfil de idosos encaminhados para o serviço de fonoaudiologia de uma Unidade Básica de Saúde (UBS) de Porto Alegre. MÉTODOS: Foram analisados os prontuários de todos pacientes do setor de fonoaudiologia da UBS, que incluía pacientes desligados, em espera ou em atendimento. Selecionou-se para o estudo apenas aqueles com idade maior ou igual a 60 anos e que possuíam prontuário completo. Foram então descritos dados demográficos e sobre o encaminhamento. RESULTADOS: Incluiu-se 21 idosos, 13 (61,9\%) do sexo feminino com idade média de 71,3 anos, e 8 (38\%) do sexo masculino com idade média de 71,5 anos. O principal motivo de encaminhamento foi a dificuldade de linguagem, seguida de disfagia, e disartria. $\bigcirc$ profissional responsável pelo maior número de encaminhamentos foi o médico clínico geral. A principal doença de base foi o Acidente Vascular Encefálico. CONCLUSÃO: Houve maior prevalência de idosos do sexo feminino, com queixa de linguagem, decorrente de AVC e encaminhados pelo clínico geral. O entendimento deste perfil populacional possibilitará a criação de estratégias para melhor atender esta demanda. 


\title{
Mobilidade e força em idosos institucionalizados: uma avaliação multiprofissional
}

\author{
Michelle Bertoglio', Lidiane Requia Alli-Feldmann², Paulo Roberto Cardoso Consoni ${ }^{3}$, \\ Jonathas da Silva Moraes ${ }^{4}$, Lucimara Pereira ${ }^{5}$, Kelly Diana Pereira da Cruz \\ Daniel da Rosa Vieira ${ }^{7}$, Jadiane Fucilini Martins ${ }^{8}$
}

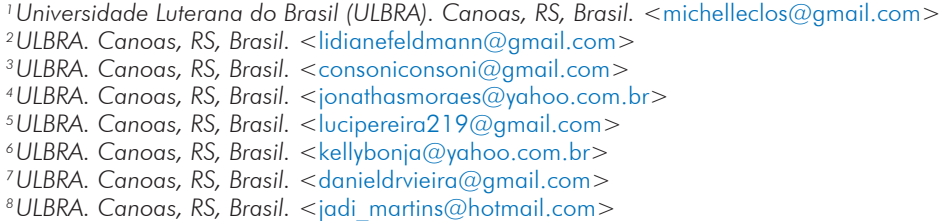

INTRODUÇÃO: $\bigcirc$ envelhecimento é um fenômeno presente em nossa sociedade, assim como o crescente número de idosos residentes em Instituições de Longa Permanência. A condição de saúde de idosos institucionalizados tende a apresentar características diferenciadas dos idosos não institucionalizados, em parte influenciada pela redução da rede de suporte social, acesso precarizado a serviços e tratamentos adequados de saúde. Isto nos sinaliza sobre a importância de acompanhamento sistemático desta população nos quesitos mobilidade, funcionalidade e fragilidade. Portanto, o presente trabalho foi desenvolvido por docentes e acadêmicos vinculados ao Núcleo de Atenção Geronto-Geriátrica da Ulbra/Canoas (NEAGG), das áreas de educação física, enfermagem, medicina, biomedicina, farmácia, fonoaudiologia e serviço social. Tem por objetivo avaliar a força de preensão palmar e a mobilidade de idosos residentes em uma ILPI, de caráter social, na cidade de Canoas. A amostra foi composta por 20 idosos, com média de 77,2 anos, de ambos os sexos. Para membros superiores foram avaliados a força de preensão palmar, através de dinamômetro digital marca Instrutherm, para avaliar a mobilidade de membros superiores utilizou-se o teste de alcançar as costas proposto por Rikli e Jones (2008), e para avaliar o risco de quedas foi utilizado o teste "Timed Up and Go" (TUG). As análises de dados foram realizadas através do software estatístico SPSS, versão 25.0, verificando média e desvio padrão. Os resultados encontrados mostraram a força de preensão com uma média de 14,31 $\pm 7,43 \mathrm{kgf}$; a mobilidade dos ombros ficou com média de $-27 \pm 16,56 \mathrm{~cm}$. Relacionado ao risco de quedas a média do grupo foi de $23,74 \pm 17,17$ segundos. Estes resultados indicam perda de mobilidade articular dos ombros e maior risco de quedas potencializando o risco de morbidades crônicas, distúrbios musculoesqueléticos e fator de risco para síndrome de fragilidade, assim como escore negativo em testes de flexibilidade são sugestivos para perda na amplitude de movimento articular e consequentemente mobilidade. Sugere-se que a prática de atividades físicas regulares e de reabilitação implementariam ganhos na mobilidade funcional, força e estabilidade postural dos idosos institucionalizados. 


\title{
Relação de idosas institucionalizadas com seus familiares através das tecnologias como aliadas na manutenção dos vínculos
}

\author{
Ariela Pinto Quartiero', Daniela Trevisan Monteiro², Aline Cardoso Siqueira ${ }^{3}$ \\ 'Universidade Federal de Santa Maria (UFSM). Santa Maria, RS, Brasil. <arielaquartiero@gmail.com> \\ 2UFSM. Santa Maria, RS, Brasil.<daniela.trevisan.monteiro@gmail.com> \\ ${ }^{3}$ UFSM. Santa Maria, RS, Brasil. <alinecsiq@gmail.com>
}

INTRODUÇÃO: O processo de envelhecimento é único e singular para cada sujeito e é natural que as necessidades aumentem com o passar dos anos, demandando muitas vezes cuidados específicos. Por esse motivo, alguns idosos optam, ou são enviados, a residir em instituições de longa permanência que ofereçam manejos adequados. $O$ que ocorre, em alguns casos, é o fato dos familiares não realizarem a visita ao idoso na instituição. OBJETIVOS: Este trabalho tem como objetivo apresentar e discutir o uso das novas tecnologias, como Facebook e WhatsApp no resgate e na manutenção dos vínculos familiares. MÉTODO: O trabalho ocorre em uma instituição de longa permanência para mulheres idosas, no interior do Rio Grande do Sul. Nesta, residem cerca de 200 mulheres com idade de 60 anos ou mais. Desde a implantação do setor de Psicologia, em 2011 , existe uma demanda que se repete - a ausência dos familiares nas visitas às idosas institucionalizadas. Frente a esta situação recorrente, uma alternativa, para diminuir o sofrimento da distância entre os parentes, foi manter os vínculos através da utilização do WhatsApp e Facebook. Essa aproximação acontece durante os atendimentos psicológicos semanais, a partir da demanda das idosas de querer informações e contato com seus familiares. Esse contato é agendado no dia anterior ao atendimento e, no dia da sessão, o familiar entra em contato através dos aplicativos. RESULTADOS: Observou-se, desde então, uma maior adaptação das senhoras que conseguem estabelecer essa relação, mesmo que virtual, indicando uma diminuição da ansiedade e um maior envolvimento nas participações de atividades dentro da instituição, desenvolvendo sua autonomia e empoderamento nas suas relações. CONCLUSÕES: Considera-se que os vínculos familiares são fundamentais para preservar a saúde mental das idosas, entendendo que esses vínculos não necessariamente se dão de forma presencial, mas, igualmente, virtual, assegurando-Ihes o direito de sentirem-se próximas dos seus familiares. 


\title{
Atenção ao idoso portador de Transtorno Mental a partir da visita domiciliar
}

\section{Evelyn de Cassia Pereira Costa ${ }^{1}$, Ana Paula Brum Müller², Bruna Barros Rezende Bellote ${ }^{3}$, Giovanna Capinzaiki de Macedo ${ }^{4}$}

Universidade Estácio de Sá. Porto Alegre, RS, Brasil. <ecpcosta@yahoo.com.br>

2Universidade Estácio de Sá. Porto Alegre, RS, Brasil.<anapbmuller@outlook.com>

3Universidade Estácio de Sá. Porto Alegre, RS, Brasil. < bruna.beloti@hotmail.com>

${ }^{4}$ Universidade Estácio de Sá. Porto Alegre, RS, Brasil. <gi capinzaiki@hotmail.com>

\begin{abstract}
INTRODUÇÃO: Transtornos mentais acometem a população em geral e, dentre os idosos, um terço deles apresentam algum Transtorno Mental. Atualmente, é preconizado pelas Políticas de Atenção à Saúde o cuidado integral e humanizado aos usuários dos serviços de saúde. Para isso, existe a possibilidade de inserção na Estratégia de Saúde da Família, que apresenta, como uma das suas principais ferramentas, a realização da visita domiciliar por uma equipe de saúde. Essa ação torna-se importante principalmente àqueles que apresentam dificuldades no deslocamento. OBJETIVO: Dentro desta perspectiva, o objetivo deste trabalho é descrever a experiência de visita domiciliar à uma paciente idosa com Transtorno Mental, em tratamento por um serviço público ambulatorial, realizada por alunas da graduação em Medicina, durante a disciplina de Saúde da Família. MÉTODO: Foi utilizada a pesquisa qualitativa do tipo estudo de caso, ocorrido durante dois meses, com uma pessoa idosa, sexo feminino, 81 anos de idade, viúva, residindo sozinha, na cidade do Rio de Janeiro-RJ, com diagnóstico CID F32.2. RESULTADOS: Durante a realização das visitas domiciliares, foi verificado o uso de medicação, apoio familiar, condições socioeconômicas, condições físicas e psíquicas do paciente e outras demandas solicitadas pelos profissionais da equipe de saúde e/ou famílias. Através das visitas, conseguiu-se aprimorar o plano terapêutico e seu familiograma, levantaram-se os pontos positivos que facilitavam a adesão do paciente ao tratamento e os pontos negativos que devem ser trabalhados para prevenir recaídas ou complicações, obtendo melhora significativa dos sintomas. CONCLUSÃO: Esta experiência reafirmou que a visita domiciliar pode ser uma aliada para planejar as ações de assistência ao paciente por possibilitar a aproximação da realidade familiar e social do usuário. Dificuldades relacionadas ao serviço, à rede de atenção pública, ao usuário e seus familiares também foram detectadas.
\end{abstract}




\title{
Percepção dos prescritores sobre medicamentos potencialmente inapropriados para idosos
}

\author{
Paula Thomé Dalbem', Taiane Santos Garcia², Isabela Heineck ${ }^{3}$ \\ 'Universidade Federal do Rio Grande do Sul (UFRGS). Porto Alegre, RS, Brasil. <paula dalbem@hotmail.com> \\ 2UFRGS. Porto Alegre, RS, Brasil. <taiane.sg@gmail.com> \\ ${ }^{3}$ UFRGS. Porto Alegre, RS, Brasil. <isabela.heineck@ufrgs.br>
}

\begin{abstract}
INTRODUÇÃO: Medicamentos que não têm indicação baseada em evidência e podem causar mais danos ao idoso do que benefícios são denominados medicamentos potencialmente inapropriados para idosos (MPIs). OBJETIVOS: Obter informações sobre a percepção dos prescritores em relação a cuidados na prescrição de medicamentos para idosos e analisar o conhecimento destes em relação à critérios explícitos de classificação de MPIs. MÉTODOS: Sob aprovação do Comitê de Ética do Hospital de Clínicas de Porto Alegre (parecer no 2.466.939), foi aplicado um questionário anônimo, através dos formulários do Google ${ }^{\circledR}$, com oito questões a 20 prescritores da UBS Santa Cecília (Porto Alegre/RS). RESULTADOS: Dos 20 profissionais que responderam ao questionário $45 \%$ eram professores, 35\% médicos residentes e $20 \%$ médicos contratados. Em relação aos idosos, $70 \%$ dos prescritores percebem boa adesão ao tratamento; e 40\% maior frequência de reações adversas a medicamentos, quando comparados à população em geral. Somente 30\% relataram conhecimento sobre algum critério de classificação de MPls, e $25 \%$ desta parcela já utilizou/utiliza algum dos critérios na prática clínica. Porém, citaram as classes mais presentes no Critério de Beers para MPIs como candidatas a desprescrição e ajuste de dose. CONCLUSÕES: $O$ conhecimento e aplicação de critérios de classificação de MPIs na prática clínica é ainda incipiente, mesmo em Unidade vinculada a Hospital Universitário. No entanto, percebe-se um cuidado maior ao prescrever medicamentos para idosos, sugerindo que uma parcela significativa de MPls está sendo monitorada adequadamente.
\end{abstract}




\title{
Dados epidemiológicos sobre o suicídio (CID 10, X60-X84) entre idosos no estado do Rio Grande do Sul, Brasil (2011-2016)
}

\author{
Ferdinando Agostinho', Karoline Monteiro de Oliveira Cardoso², Maria Luiza DÁvila Sandri', \\ Ondina Almeida Resende ${ }^{4}$, Franciely dos Passos Pereira ${ }^{5}$, Karlla Kristinna Almeida Medeiros 6 , \\ Camila Botelho Miguel ${ }^{7}$, Wellington Francisco Rodrigues ${ }^{8}$
}

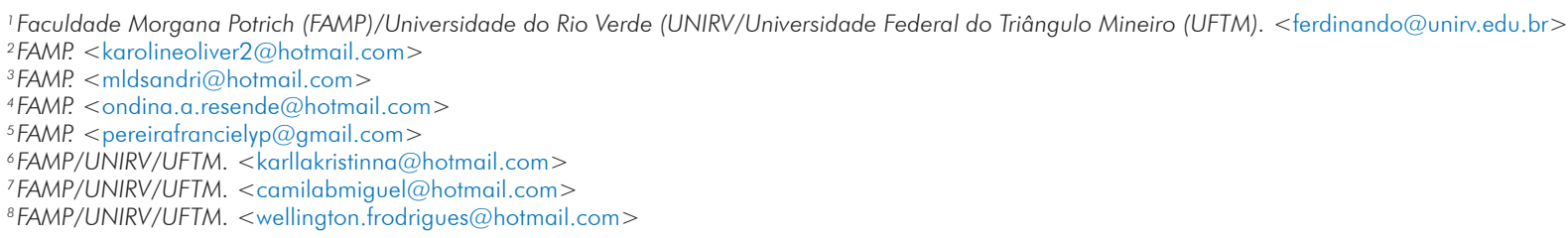

\begin{abstract}
INTRODUÇÃO: O suicídio é uma das principais causas de morte no mundo e um importante problema de saúde pública. Entre idosos, a principal causa de suicídio é a presença de depressão. OBJETIVOS: $O$ estudo objetivou descrever a taxa de incidência de casos de suicídios entre idosos no estado do Rio Grande do Sul, estratificados por faixa etária e categoria do CID 10. MÉTODO: Foram utilizados dados do IBGE/CENSO-2010 e SIM/Datasus, do qual foram selecionadas as categorias do CID10 (X60-X84) no período de 2011 a 2016. A taxa de incidência (TI) foi calculada para duas faixas etárias: $\leq 59$ e $\geq 60$ anos, utilizando a fórmula (número de casos registrados no período $\times 10.000 /$ população). RESULTADOS: Para o período, foram identificados 6.786 casos de suicídio, destes, aqueles envolvendo idosos representaram 26,71\% (1.813), neste grupo, a incidência de casos foi maior entre os homens $(80,9 \%)$ e a categorias do CID 10 com maior incidência de casos foi X70 "Lesao autoprovocada intencionalmente por enforcamento, estrangulamento ou sufocamento" (1.311 casos (72,31\%)). A TI de mortes por suicídio na população em geral foi de 6,35 (p/10.000 habitantes (hab)); para idosos ( $\geq 60$ anos), a Tl foi de 12,42 $(\mathrm{p} / 10.000 \mathrm{hab})$ versus $5,38(\mathrm{p} / 10.000 \mathrm{hab})$ da faixa etária $\leq 59$ anos. Para idosos do sexo masculino a categoria com maior incidência de casos foi "X70" (1 .055 casos (72\%)), a TI foi de 23,40 (p/10.000 hab) versus 8,53 ( $\mathrm{p} / 10.000$ hab) da faixa etária $\leq 59$ anos. Para as idosas a categoria do CID 10 com maior incidência de casos também foi "X70" (256 casos (45,39\%)), a TI foi de 4,15 (p/10.000 hab) versus 2,28 (p/10.000 hab) da faixa etária $\leq 59$ anos. CONCLUSÃO: Pode-se concluir que a taxa de incidência de mortes ocasionadas por suicídio entre idosos é maior que a de outras faixas etárias, possuindo características específicas entre os sexos. Evidencia-se que o suicídio é um problema de saúde pública e deve ser obrigatoriamente considerado em Gerontologia e Geriatria.
\end{abstract}




\section{Dados epidemiológicos sobre o suicídio (CID 10, X60-X84) entre idosos no estado de Santa Catarina, Brasil (2011-2016)}

\section{Ferdinando Agostinho', Karoline Monteiro de Oliveira Cardoso², Maria Luiza DÁvila Sandri', Ondina Almeida Resende ${ }^{4}$, Franciely dos Passos Pereira ${ }^{5}$, Karlla Kristinna Almeida Medeiros 6 , Camila Botelho Miguel ${ }^{7}$, Wellington Francisco Rodrigues ${ }^{8}$}

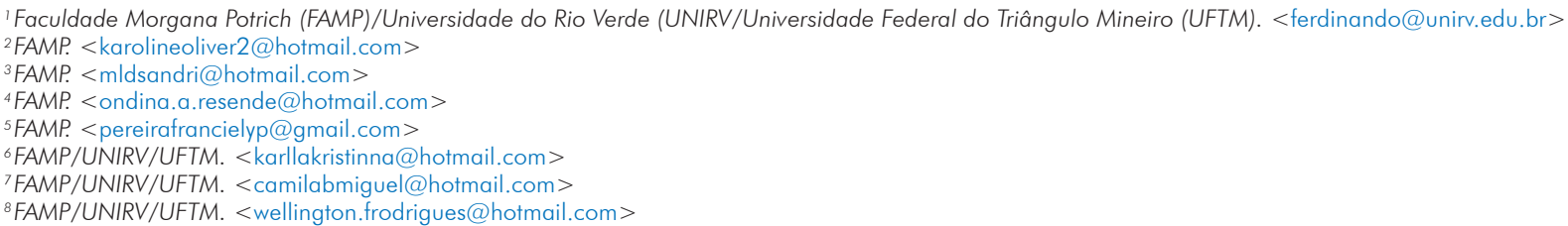

INTRODUÇÃO: O suicídio é uma das principais causas de morte no mundo e um importante problema de saúde pública. Entre idosos, a principal causa de suicídio é a presença de depressão. OBJETIVOS: Este estudo objetivou descrever a taxa de incidência de casos de suicídios entre idosos no estado de Santa Catarina, estratificados por faixa etária e categoria do CID 10. MÉTODO: Foram utilizados dados do IBGE/CENSO2010 e SIM/Datasus na qual foram selecionadas as categorias do CID10 (X60-X84) no período de 2011 a 2016. A taxa de incidência (TI) foi calculada para duas faixas etárias: $\leq 59$ e $\geq 60$ anos, utilizando a fórmula (número de casos registrados no período x 10.000/população). Resultados: Foram identificados 3.534 casos de suicídio destes, aqueles envolvendo idosos representaram 20,54\% (726), neste grupo, a incidência de casos foi maior entre os homens $(79,89 \%)$ e a categorias do CID 10 com maior incidência de casos foi X70 "Lesão autoprovocada intencionalmente por enforcamento, estrangulamento ou sufocamento" (554 casos (76,30\%)). A TI de mortes por suicídio na população em geral foi de 5,66 (p/10.000 habitantes (hab)); para idosos ( $\geq 60$ anos), a TI foi de 11,05 (p/10.000 hab) versus 5,02 ( $/ 10.000$ hab) da faixa etária $\leq 59$ anos. Para idosos do sexo masculino a categoria com maior incidência de casos foi "X70" (450 casos $(77,58 \%))$, a TI foi de 19,65 (p/10.000 hab) versus 7,63 (p/10.000 hab) da faixa etária $\leq 59$ anos. Para as idosas a categoria do CID 10 com maior incidência de casos também foi "X70" (104 casos (71,23\%)), a TI foi de 4 (p/10.000 hab) versus 2,39 (p/10.000 hab) da faixa etária $\leq 59$ anos. CONCLUSÃO: Pode-se concluir que a taxa de incidência de mortes ocasionadas por suicídio entre idosos é maior que a de outras faixas etárias, possuindo características específicas entre os sexos. Evidencia-se que o suicídio é um problema de saúde pública e deve ser obrigatoriamente considerado em Gerontologia e Geriatria. 


\section{Mortalidade por SRAG causada por Influenza em idosos no RS entre 2013-2017}

\section{Pedro Pablo de Gusmão Bonilla', Andrea Morais de Gusmão}

'R7 MFC GHC. <bonilla.pp@gmail.com>

2MFC. <amdgusmao@gmail.com>

INTRODUÇÃO: As infecções respiratórias constituem um conjunto de doenças comumente relacionadas aos idosos, sendo o vírus da Influenza um dos seus principais agentes etiológicos. Os idosos são um grupo vulnerável porque a idade avançada está associada com uma grande prevalência de doenças crônicas e sistema imune mais deficiente. No Brasil, a política de vacinação contra a Influenza iniciou-se em 1999, sendo oferecida anualmente pelo SUS aos grupos de risco. OBJETIVOS: Comparar a mortalidade por SRAG causada por Influenza entre os idosos e os demais grupos de riscos e entre os idosos vacinados e não vacinados no RS durante os últimos 5 anos. MÉTODOS: Estudo transversal com análise secundária dos dados presentes nos boletins epidemiológicos do Centro Estadual de Vigilância em Saúde (CEVS) de 2013-2017. RESULTADOS: No período entre 2013 e 2017, no Rio Grande do Sul, houve 2.629 casos de SRAG por Influenza, dos quais, 22,8\% (600 casos) foram em adultos maiores que 60 anos. Além disso, dos 368 óbitos, 141 foram em idosos, representando 38,3\% do total. O percentual de mortalidade nos idosos foi 2 vezes maior que os demais grupos de risco e entre os idosos, aqueles não vacinados apresentaram um risco 1,25 vezes maior para o óbito. CONCLUSÕES: Durante o período analisado, os idosos foram o grupo com a maior mortalidade por complicações respiratórias causadas pela Influenza. A vacina é um fator protetor importante, diminuindo o percentual de óbitos naqueles idosos que foram vacinados. 


\section{Dados epidemiológicos sobre o suicídio (CID 10, X60-X84) entre idosos no estado do Paraná, Brasil (2011-2016)}

\section{Ferdinando Agostinho', Karoline Monteiro de Oliveira Cardoso², Maria Luiza DÁvila Sandri', Ondina Almeida Resende ${ }^{4}$, Franciely dos Passos Pereira ${ }^{5}$, Karlla Kristinna Almeida Medeiros 6 , Camila Botelho Miguel ${ }^{7}$, Wellington Francisco Rodrigues ${ }^{8}$}

'Faculdade Morgana Potrich (FAMP)/Universidade do Rio Verde (UNIRV/Universidade Federal do Triângulo Mineiro (UFTM).<ferdinando@unirv.edu.br> 2FAMP.<karolineoliver2@hotmail.com>

${ }^{3}$ FAMP. < mldsandri@hotmail.com>

4FAMP.<ondina.a.resende@hotmail.com>

5FAMP < pereirafrancielyp@gmail.com>

${ }^{6}$ FAMP/UNIRV/UFTM. < karllakristinna@hotmail.com>

TFAMP/UNIRV/UFTM. < camilabmiguel@hotmail.com>

${ }^{8}$ FAMP/UNIRV/UFTM. <wellington.frodrigues@hotmail.com>

INTRODUÇÃO: O suicídio é uma das principais causas de morte no mundo e um importante problema de saúde pública. Entre idosos, a principal causa de suicídio é a presença de depressão. OBJETIVOS: Este estudo objetivou descrever a taxa de incidência de casos de suicídios entre idosos no estado de Paraná, estratificados por faixa etária e categoria do CID 10. MÉTODO: Foram utilizados dados do IBGE/CENSO2010 e SIM/Datasus, do qual foram selecionadas as categorias do CID10 (X60-X84) no período de 2011 a 2016. A taxa de incidência (TI) foi calculada para duas faixas etárias: $\leq 59$ e $\geq 60$ anos, utilizando a fórmula (número de casos registrados no período x 10.000/população). RESULTADOS: Para o período, foram identificados 3.973 casos de suicídio destes, aqueles envolvendo idosos representaram 16,23\% (645), neste grupo, a incidência de casos foi maior entre os homens $(84,80 \%)$ e a categorias do CID 10 com maior incidência de casos foi X70 "Lesao autoprovocada intencionalmente por enforcamento, estrangulamento ou sufocamento" (431 casos $(66,82 \%))$, a TI de mortes por suicídio na população em geral foi de 3,80 (p/ 10.000 habitantes (hab)); para idosos ( $\geq 60$ anos), a TI foi de 5,51 (p/10.000 hab) versus 3,58 (p/10.000 hab) da faixa etária $\leq 59$ anos. Para idosos do sexo masculino a categoria com maior incidência de casos foi "X70" (372 casos $(68,01 \%))$, a TI foi de 10,19 (p/10.000 hab) versus 5,73 (p/10.000 hab) da faixa etária $\leq 59$ anos. Para as idosas a categoria do CID 10 com maior incidência de casos também foi "X70" (59 casos (60,20\%)), a TI foi de 1,55 (p/10.000 hab) versus 1,47 $(\mathrm{p} / 10.000 \mathrm{hab})$ da faixa etária $\leq 59$ anos. CONCLUSÃO: Pode-se concluir que a taxa de incidência de mortes ocasionadas por suicídio entre idosos é maior que a de outras faixas etárias, possuindo características específicas entre os sexos. Evidencia-se que o suicídio é um problema de saúde pública e deve ser obrigatoriamente considerado em Gerontologia e Geriatria. 


\title{
Aspectos sociodemográficos e clínicos de idosas com e sem incontinência urinária atendidas em unidades básicas de saúde do município de Uruguaiana/RS
}

\author{
Ângela Kemel Zanella' \\ 'Docente do Curso de Fisioterapia da Universidade Federal do Pampa. Uruguaiana, RS, Brasil. <angelakemelzanella@gmail.com>
}

\begin{abstract}
INTRODUÇÃO: No envelhecimento o processo fisiológico predispõe o idoso ao aparecimento de doenças crônicas não transmissíveis que causam o declínio das atividades fisiológicas. Dentre as doenças que mais acometem os idosos está a incontinência urinária (IU). OBJETIVO: O trabalho teve como objetivo conhecer o perfil sociodemográfico e clínico das idosas com e sem queixa de IU atendidas nas Unidades Básicas de Saúde do município de Uruguaiana/RS. MÉTODO: A pesquisa avaliou idosas com mais de 60 anos. $O$ estudo foi do tipo transversal, analítico e descritivo. Aplicou-se a Ficha de Avaliação e Avaliação Funcional do Assoalho Pélvico (AFA). Foi utilizado o pacote estatístico SPSS versão 20.0 for Windows. RESULTADOS: Este estudo evidenciou 66 idosas continentes e 63 idosas incontinentes, de um total de 129 participantes. Segundo as características da amostra quanto às médias de idade, idade da menarca, idade da primeira relação sexual e idade da menopausa das idosas avaliadas de acordo com a perda urinária, observamos que as idosas continentes possuíram uma média de idade da menopausa, menor que as incontinentes. Em contrapartida a média de parto vaginal nas idosas continentes foi maior do que nas idosas incontinentes. Para as variáveis idade da menopausa, consumo de chocolate e chimarrão, e para os resultados da palpação bi digital foi observado diferença estatística entre os grupos. Na AFA, observou-se que as idosas do grupo incontinente apresentaram um grau de força da MAP menor que o grupo continente, sendo que, haviam idosas do grupo continente que, também, apresentaram fraqueza da MAP. CONCLUSÕ̃ES: $\bigcirc$ presente estudo constatou que a incontinência urinária tem impacto na vida da maioria das idosas, através das limitações fisiológicas impostas pela doença, de acordo com a pontuação dos escores dos domínios avaliados, quanto às características sociodemográficas, média de idade, fatores de risco, doenças crônicas, consumo de alimentos e bebidas, palpação bi digital entre as idosas continentes e incontinentes.
\end{abstract}




\title{
Comparação entre os sexos feminino e masculino quanto a percepção da morte por médicos residentes e estudantes de Medicina
}

\author{
Thalita Felsky dos Santos', Rafael Sonoda Akamine' ${ }^{2}$, Vitor Last Pintarelli ${ }^{3}$ \\ Universidade Federal do Paraná (UFPR). <thalitafelsky@gmail.com> \\ 2UFPR.<nkcrafael@gmail.com> \\ 3UFPR.<vitorpintarelli@yahoo.com.br>
}

\begin{abstract}
INTRODUÇÃO: Considerando o estresse emocional que a morte traz a estudantes de medicina (EM) e a médicos residentes (MR), é surpreendente que exista um número limitado de estudos quanto ao tema. OBJETIVOS: Avaliar a percepção do EM do Curso de Medicina da Universidade Federal do Paraná (CM-UFPR) e MR do Hospital de Clínicas da UFPR (HC-UFPR) sobre o morrer e a morte, com base nos sexos feminino (F) e masculino (M). MÉTODOS: Estudo transversal observacional baseado no preenchimento de questionário autoaplicado sobre o morrer e a morte, inspirado no Frommelt Attitude Toward Care of the Dying Scale (FATCOD-B Scale), a EM dos 12 períodos do CM, e a MR de áreas clínico-cirúrgicas. Foi usado o teste exato de Fisher ou o teste Qui-quadrado. Valores de $p<0,05$ indicaram significância estatística. RESULTADOS: Foram avaliados 805 EM, matriculados no primeiro semestre de 2016 e 93 MR, matriculados no ano de 2016 . Analisando o sentimento de frustração e impotência após a morte de paciente para EM, foi encontrada pouca diferença entre os sexos $M$ e $F$ (EM: $50,6 \%$ do sexo $M$ e $65,9 \%$ do sexo $F(p=0,026)$; MR: 45,9\% e 66,7\%, respectivamente $(p=0,247))$. Analisando a pergunta "Eu ficaria desconfortável ao falar sobre a morte iminente com uma pessoa que esteja morrendo", foi constatada leve diferença entre os sexos $M$ e $F$ no grupo dos $\operatorname{EM}(51,6 \%$ do sexo $M$ e $59,8 \%$ do sexo $F(p=0,013)$ ); no grupo dos $M R$, a diferença foi maior [15,8\% do sexo $M$ e $43,6 \%$ do sexo $F(p=$ não aplicável)]. CONCLUSÕES: É notável a existência de diferenças na percepção quanto o morrer e a morte entre os sexos $M$ e $F$ nos grupos de EM e MR no processo de formação médica. Estudos que busquem justificar os resultados encontrados neste trabalho são de extrema importância para o entendimento do tema em questão.
\end{abstract}




\title{
Jogos lúdicos adaptados: uma possibilidade terapêutica para cognição e socialização de pessoas idosas
}

\author{
Caroline Gimenez Covatti ${ }^{1}$, Aline da Costa Gobbi' ${ }^{2}$, Victória Satiagraha Weirich Soriano ${ }^{3}$, \\ Paulo Roberto Cardoso Consoni ${ }^{4}$, Michelle Bertoglio $\mathrm{Clos}^{5}$ \\ Acadêmica do curso de Medicina, Universidade Luterana do Brasil (ULBRA) - Ligante da LAGGE/ULBRA. Canoas, RS, Brasil. \\ <carolinecovatti@hotmail.com> (correspondente) \\ ${ }^{2}$ Acadêmica do curso de Medicina, ULBRA - Ligante da LAGGE/ULBRA. Canoas, RS, Brasil. <alinec.gobbi@gmail.com> \\ ${ }^{3}$ Acadêmica do curso de Medicina, ULBRA - Ligante da LAGGE/ULBRA. Canoas, RS, Brasil. <satiagrahavic@outlook.com> \\ ${ }_{4}^{4}$ Professor e coordenador da Liga Acadêmica de Geriatria e Gerontologia (LAGGE/ULBRA). Canoas, RS, Brasil. <consoniconsoni@gmail.com> \\ ${ }^{5}$ Professora e coordenadora da LAGGE/ULBRA. Canoas, RS, Brasil.<michelleclos@gmail.com>
}

INTRODUÇÃO: As Instituições de Longa Permanência (ILPIs) são uma realidade dos idosos dependentes para atividades básicas da vida diária (AVD), ou atividades cognitivas, assim como para aqueles que não possuem rede de suporte social capaz de ampará-los em suas crescentes necessidades. No entanto, é sabido que o ambiente institucionalizado pode impactar no funcionamento cognitivo se for um espaço que não oferte atividades de estimulação e socialização aos seus residentes. OBJETIVO: Analisar o impacto da atividade lúdica com jogo de dominó adaptado junto a idosos institucionalizados na cidade de Canoas, realizada por integrantes da Liga Acadêmica de Geriatria e Gerontologia da ULBRA (LAGGE/ULBRA). METODOLOGIA: A participação dos idosos, residentes na ILPI de Canoas, foi voluntária com início e término conforme motivação. O jogo proposto foi o dominó, confeccionado por ligantes da LAGGE. As peças tiveram alteração de tamanho para facilitar o manuseio e foram combinadas cores e números para que participassem idosos com menor capacidade cognitiva ou visual. RESULTADOS: Os idosos mostraram-se receptivos e motivados para o jogo. 80\% do total dos residentes puderam jogar, devido ao aumento do tamanho das peças e a distinção em cores. Os idosos recordaram as regras do jogo e não demonstraram dificuldades na atividade. O jogo lúdico dominó foi considerado relevante para a integração dos idosos, sobretudo pela adaptação de suas peças. CONCLUSÃO: Através do ato de jogar o indivíduo utiliza suas habilidades motoras e psíquicas, tornando-o consciente das suas decisões, exercitando a criatividade, estabelecendo afinidades e troca de experiências como "escutar, esperar, observar, perceber, sentir". Portanto, intervenções para melhoria das capacidades cognitivas e de socialização de idosos institucionalizados são beneficiadas com o uso de atividades lúdicas adaptadas. 


\title{
Problemas advindos do autoconceito e a autoestima de idosos: relato de experiência
}

\author{
Jarbas da Silva Ziani ', Cenir Gonçalves Tier², Ane Gabrielle Muniz ${ }^{3}$, Franciele Chervenski Morin ${ }^{4}$, \\ Muriel Sagueiro da Silva ${ }^{5}$, Maria Amanda Bibiano de Jesus ${ }^{6}$, Ariane Ferreira de Menezes ${ }^{7}$, \\ Jonatan Jean Silveira da Silva ${ }^{8}$
}

'Discente do Curso de Enfermagem. Bolsista projeto Extensão/Ensino e Pesquisa, Universidade Federal do Pampa (UNIPAMPA). <iarbas ziani@outlook.com> ${ }^{2}$ Enfermeira. Docente do Curso de Enfermagem. Orientadora do Projeto Envelhecer com Arte e Saúde, UNIPAMPA. Membro do Grupo de Estudos e Pesquisa em Enfermagem da Fronteira Oeste do Rio Grande do Sul (GEPEN-FORS). <cgtier@hotmail.com>

${ }^{3}$ Discente do Curso de Enfermagem. Bolsista projeto Extensão/Ensino e Pesquisa; UNIPAMPA.<ane.gabrielemuniz@gmail.com>

${ }^{4}$ Discente do Curso de Enfermagem. Bolsista projeto Extensão/Ensino e Pesquisa; UNIPAMPA. <francielemorin@gmail.com>

${ }_{5}^{5}$ Discente do Curso de Enfermagem. Voluntária projeto Extensão/Ensino e Pesquisa, UNIPAMPA. <muri.salgueiro@gmail.com>

${ }^{6}$ Discente do Curso de Enfermagem. Voluntária projeto Extensão/Ensino e Pesquisa, UNIPAMPA. <amandabj2008@hotmail.com>

7 Discente do Curso de Enfermagem. Voluntária projeto Extensão/Ensino e Pesquisa, UNIPAMPA. <Ariane.m.f001@gmail.com>

${ }^{8}$ Enfermeiro. Técnico em Assuntos Educacionais, UNIPAMPA. <jonatanjssilva@gmail.com>

INTRODUÇÃO: Entre os agravos que acometem idosos, destaca-se a depressão, a qual envolve aspectos biológicos, psicológicos, e pode ser responsável pela perda de autonomia, e outros agravamentos. (RIBEIRO et al., 2017). Então, entende-se que a autoestima baixa e adversidades são fatores contribuintes no processo de aparecimento de problemas psicológicos no idoso. OBJETIVO: Conhecer quais fatores podem causar problemas psicológicos em idosos. MÉTODO: Relato de experiência advindo de uma atividade desenvolvida pelo projeto de Extensão Envelhecer com Arte e Saúde em uma Estratégia de Saúde da Família do Município do Rio Grande do Sul. Dentre as ações desenvolvidas com os idosos, destaca-se a "Refletindo sobre os problemas psicológicos que acometem a pessoa idosa", por meio de uma roda de conversa. Apresentaram-se as principais doenças psicológicas que acometem os idosos. O projeto está registrado no SIPPEE da Unipampa sob no 10.065.16. RESULTADO: Participaram desta atividade cinco idosos, com idades entre 65 a 75 anos. Diante dos relatos pode-se compreender que o principal obstáculo enfrentado pelos idosos é a relação com os seus familiares, a falta de identidade e autoestima baixa os quais podem ocasionar depressão nestes. $O$ sentimento de abandono que eles sentem por parte dos seus familiares tornaos frágeis emocionalmente. A autoestima está correlacionada com os resultados de vida, dentre eles as relações humanas, o trabalho, a saúde e, consequentemente, o envelhecimento saudável (TAVARES et al., 2016). Conseguintemente idosos que possuem um bom relacionamento tem uma autoestima mais elevada, menor suscetibilidade a problemas psicológicos e um envelhecimento mais sadio. CONCLUSÃO: Torna-se imprescindível ver o idoso como um ser holístico, compreendendo todas as necessidades que o mesmo precisa para desenvolver um envelhecimento ativo e saudável e evitar o surgimento de problemas psicológicos. 


\title{
Associação entre consumo de proteína animal, circunferências e força muscular em nonagenários e centenários: AMPAL
}

\author{
Luana de Souza Goulart', Renata Breda Martins², Raquel Seibel ${ }^{3}$, Valéria Baccarin laniski ${ }^{4}$, \\ Josemara de Paula Rocha ${ }^{5}$, Ângelo José Gonçalves Bós ${ }^{6}$ \\ Nutricionista. Mestranda em Gerontologia Biomédica, Pontifícia Universidade Católica do Rio Grande do Sul (PUCRS). Porto Alegre, RS, Brasil. \\ <luana.goulart+@gmail.com> \\ 2Nutricionista. Mestranda em Gerontologia Biomédica, PUCRS. Porto Alegre, RS, Brasil.<nutri.renatamartins@gmail.com> \\ ${ }^{3}$ Nutricionista. Doutoranda em Gerontologia Biomédica, PUCRS. Porto Alegre, RS, Brasil.<raquelseibel@hotmail.com> \\ ${ }^{4}$ Nutricionista. Mestranda em Gerontologia Biomédica, PUCRS. Porto Alegre, RS, Brasil.<valeriaianiski@yahoo.com.br> \\ ${ }^{5}$ Fisioterapeuta. Doutoranda em Gerontologia Biomédica, PUCRS. Porto Alegre, RS, Brasil.<josemara.rocha@hotmail.com> \\ ${ }^{6}$ Geriatra. Doutor em Medicina, PUCRS. Porto Alegre, RS, Brasil.<angelo.bos@pucrs.br>
}

\begin{abstract}
INTRODUÇÃO: A literatura descreve o menor consumo de fontes proteicas, principalmente, de origem animal, como carnes, leites e derivados em longevos. A diminuição do consumo de proteínas tem sido associada à redução da massa e força muscular, comuns nesta faixa etária. OBJETIVO: Verificar se o consumo de proteína animal está associado a circunferências e força muscular em longevos. Métodos: Estudo transversal, realizado através de entrevista domiciliar, de junho-dezembro de 2016 . O consumo de leite e/ou derivados, carne vermelha, frango ou peixe foram categorizados entre consumo $\leq 6$ dias ou 7 dias/semana. A análise estatística incluiu média e desvio padrão, e associação foi realizada através da regressão linear, utilizando software Epi info ${ }^{\text {TM }} 7.2$. RESULTADOS: Foram avaliados 186 longevos, na maioria mulheres 73,0\% ( $n=173)$, média etária de 92,16 $\pm 3,51$ anos, média

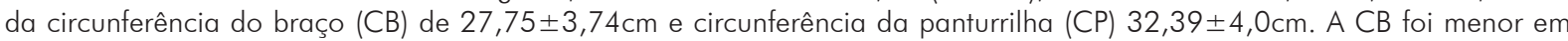
quem consome leite ou derivados 7 dias/semana $(29,11 \pm 4,0 \mathrm{~cm})$, não havendo diferenças significativas entre grupos quanto a força de preensão palmar (FPP). A CB foi maior naqueles que consomem carne vermelha 7 dias $/$ semana $(28,35 \pm 3,84 \mathrm{~cm})$,

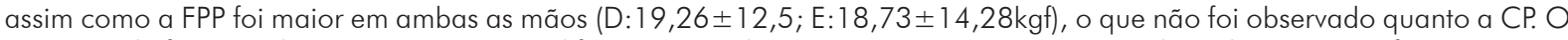
consumo de frango 7dias/semana não teve diferença em relação a CB e a CP, porém a FPP de ambas as mãos foram maiores naqueles que consomem frango diariamente ( $D: 15,43 \pm 8,8 ; E: 15,63 \pm 10,87 \mathrm{kgf}$ ). Quanto ao consumo de peixe, aqueles que não consomem diariamente apresentaram maior medidas da CB e da CP. Naqueles que não consomem peixe a FPP foi maior

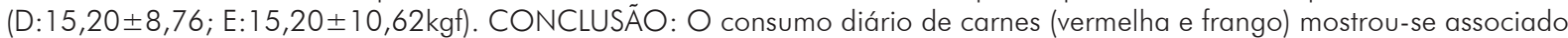
com a força muscular em longevos, o que não conseguiu ser observado com relação as circunferências. Esse estudo reforça a importância de incentivar o consumo adequado de proteína de origem animal.
\end{abstract}




\title{
Relação entre deficiência de vitamina $D$ nos idosos brasileiros e saúde na terceira idade: uma revisão sistemática
}

\section{Jéssica Vendruscolo Dos Santos' ${ }^{1}$ Vera Elizabeth Closs², Viviane Carrion Castanho ${ }^{3}$, Martine Elisabeth Kienzle Hagen ${ }^{4}$}

'Universidade Federal do Rio Grande do Sul (UFRGS). Porto Alegre, RS, Brasil.<jessicav.nutri@hotmail.com>

${ }^{2}$ Pontifícia Universidade Católica do Rio Grande do Sul (PUCRS). Porto Alegre, RS, Brasil. <veraec@terra.com.br>

3UFRGS. Porto Alegre, RS, Brasil. <00009239@ufrgs.br>

4UFRGS. Porto Alegre, RS, Brasil.<martine.hagen@ufrgs.br>

\begin{abstract}
INTRODUÇÃO: Estudos epidemiológicos têm demonstrado gradativa diminuição nos níveis de vitamina D, constituindo-se em uma epidemia mundial. Sua deficiência tem sido associada à osteoporose, ao hiperparatiroidismo secundário, hipertensão arterial, secreção hormonal anormal e resistência à insulina e está entre as carências mais frequentes entre os idosos. Alguns estudos têm apresentado elevada hipovitaminose D no Brasil, em cenários clínicos específicos. Porém, ainda não são conhecidos dados populacionais que descrevam sua prevalência em idosos. OBJETIVO: Revisão sistemática da prevalência da deficiência de vitamina $D$ em idosos no Brasil e regiões, relacionando os fatores de risco para doenças e agravos decorrentes. MÉTODO: O estudo obedece às diretrizes do Ministério da Saúde, Cochrane, PRISMA e MOOSE, para revisões sistemáticas. A formulação da questão de pesquisa foi efetuada pelo método PICo: População de Interesse (idosos com $\geq 60$ anos), Interesse (prevalência de hipovitaminose D) e Contexto (Brasil e macrorregiões). A busca explorou plataformas eletrônicas MEDLINE (via PUBMED), EMBASE, SCIELO e LILACS (via Biblioteca Virtual de Saúde), utilizando-se descritores MeSH, descritores em ciências da saúde (DECs) e indexadores emtrees. Foram incluídos os estudos observacionais, publicados até outubro de 2017, com idosos (ambos os sexos) do Brasil, nos idiomas inglês, espanhol ou português. A revisão foi submetida à plataforma PROSPERO no 74732. RESULTADOS: Foram selecionados 758 estudos, destes 561 para leitura do resumo e 216 para leitura completa. Por fim foram incluídos na análise 32 estudos, 71,9\% com delineamento transversal, 15,6\% de coorte e 12,5\% de caso-controle. No total, foram avaliados 12.122 idosos. A prevalência de deficiência de vitamina D variou de 19,2\% a 98,2\% (média 61,1\%). CONCLUSÃO: Os dados parciais revelam a elevada prevalência de hipovitaminose D em idosos, este estudo permitirá conhecer dados epidemiológicos no Brasil e regiões.
\end{abstract}




\title{
Medicamentos potencialmente inapropriados para idosos em uma Unidade Básica de Saúde de Porto Alegre
}

\author{
Taiane Santos Garcia', Paula Thomé Dalbem², Bruno Simas da Rocha ${ }^{3}$, Isabela Heineck \\ 'Universidade Federal do Rio Grande do Sul (UFRGS). Porto Alegre, RS, Brasil.<itaiane.sg@gmail.com> \\ 2 UFRGS. Porto Alegre, RS, Brasil. < paula dalbem@hotmail.com> \\ ${ }^{3}$ Hospital de Clínicas de Porto Alegre. Porto Alegre, RS, Brasil. <bsrocha@hcpa.edu.br> \\ ${ }_{4}^{4}$ UFRGS. Porto Alegre, RS, Brasil.<isabela.heineck@ufrgs.br>
}

\begin{abstract}
INTRODUÇÃO: O elevado número de medicamentos prescritos para o idoso favorece a prescrição de medicamentos potencialmente inapropriados. Medicamentos que não têm indicação baseada em evidência, e podem causar mais danos ao idoso do que benefícios, são denominados medicamentos potencialmente inapropriados para idosos (MPIs). OBJETIVOS: Analisar a população idosa de uma Unidade Básica de Saúde (UBS), classificando pacientes em polifarmácia e o uso de MPIs. MÉTODOS: Foram consultados os prontuários de 390 idosos com 60 anos ou mais que fazem acompanhamento clínico na UBS Santa Cecília, em Porto Alegre. Foram avaliados os medicamentos referidos nos prontuários como "em uso" por cada paciente na última consulta registrada. Polifarmácia foi definida como uso de 5 medicamentos ou mais e os MPls foram classificados conforme critério de Beers (2012) e pelo sistema Anatômico Terapêutico Químico (Anatomical Therapeutic Chemical - ATC). RESULTADOS: Encontrou-se uma média de 5,3 medicamentos em uso por idoso $\pm 2,7$; onde identificou-se polifarmácia em 207 pacientes (53\%). Classificou-se 54 MPls como "em uso", onde 186 pacientes (48\%) utilizavam pelo menos 1 MPI. Do total de MPIs prescritos, destaca-se que 52,9\% eram de ação no sistema nervoso central, 13,5\% no sangue e órgãos hematopoiéticos e 10,4\% no sistema cardiovascular. CONCLUSÕES: O alto índice de idosos em uso de medicamentos potencialmente inapropriados aponta que deve-se intensificar a farmacovigilância na atenção primária, além de investir em protocolos de desprescrição e uso racional de medicamentos nesta população.
\end{abstract}




\title{
Necessidade de cuidados paliativos em pacientes com insuficiência cardíaca avançada internados em um hospital terciário
}

\author{
Roman Orzechowski ${ }^{1}$, Luciana Campos ${ }^{2}$, Emilio Hideyuki Moriguchi², \\ Roberta Rigo Dalla Corte ${ }^{2}$, Renato Gorga Bandeira de Melo²
}

'Hospital Nossa Senhora da Conceição.Porto Alegre, RS, Brasil.<romanbis@yahoo.com.br> (correspondente)

${ }^{2}$ Hospital Nossa Senhora da Conceição. Porto Alegre, RS, Brasil.

\begin{abstract}
INTRODUÇÃO: Apesar dos avanços importantes no tratamento da Insuficiência Cardíaca Avançada através do transplante cardíaco, esta continua sendo uma epidemia mundial, com alta morbidade, hospitalização recorrente e expectativa de vida menor do que cinco anos. Médicos e pacientes superestimam a sobrevida, o que torna a indicação de cuidados paliativos desafiadora. Estratégias paliativas envolvendo comunicação honesta, gerenciamento efetivo de sintomas, apoio psicológico e espiritual podem melhorar a qualidade de vida e reduzir a carga física e emocional do paciente. OBJETIVOS: Avaliar a necessidade de Cuidados Paliativos em pacientes com Insuficiência Cardíaca Congestiva Avançada internados em uma enfermaria cardiológica. MÉTODO: Aplicação da Escala Necessidades Paliativas (NECPAL) da Organização Mundial da Saúde que inclui questão surpresa com o médico assistente. Outras questões com o paciente e/ou responsável, para avaliar a indicação de Cuidados Paliativos. Foram incluídos 82 pacientes entre junho e novembro de 2017 com diagnóstico de Insuficiência Cardíaca classes III/IV ou fração de ejeção menor ou igual a $40 \%$ em ecocardiografia dos últimos 12 meses. RESULTADOS: média de idade 68 $\pm 20,51$ pacientes do sexo masculino e 31 pacientes do sexo feminino. Quarenta e três pacientes (52,4\%) eram casados ou estavam em união consensual e 10 (12\%) moravam sozinhos. O óbito de 46 pacientes $(56,1 \%)$ nos 12 meses subsequentes não surpreenderia o seu médico e quarenta e cinco pacientes (55\%), $p<0,01$ tiveram indicação de cuidados paliativos pela escala NECPAL. Desnutrição, funcionalidade, fragilidade e comorbidades, $p<0,01$ foram significativos para indicação de Cuidados Paliativos. CONCLUSÃO: Cerca de metade dos pacientes que internaram por ICC classe III-IV teriam indicação de Cuidados Paliativos. Novos estudos são necessários para o seguimento de longo prazo para avaliação da escala NECPAL em diferentes contextos de saúde da população brasileira.
\end{abstract}




\title{
Processo de enfermagem no cuidado à pessoa idosa com Diabetes Mellitus tipo 2
}

\author{
Sheila Silveira Costa', Deise Lima Righi², Rita Arim Rosales ${ }^{3}$, Janaína Amorim de Ávila ${ }^{4}$, \\ Bárbara Tarouco da Silva ${ }^{5}$, Marlene Teda Pelzer ${ }^{6}$ \\ 'Enfermeira. Mestre em Enfermagem pelo Programa de Pós-Graduação em Enfermagem da Universidade Federal do Rio Grande (PPGEnf/FURG). \\ Membro Integrante do Grupo de Estudo e Pesquisa em Gerontogeriatria, Enfermagem/Saúde e Educação (GEP-GERON/FURG/CNPq). \\ <sheilasilco@hotmail.com> (correspondente) \\ 2Enfermeira. Mestre em Enfermagem pelo PPGEnf/FURG. Membro Integrante do GEP-GERON/FURG/CNPq. <deisenfermagem@ymail.com> \\ ${ }^{3}$ Enfermeira. Doutora em Enfermagem pelo PPGEnf/FURG. Membro Integrante do GEP-GERON/FURG/CNPq. <enfaritarim@yahoo.com.br> \\ ${ }^{4}$ Enfermeira. Doutora em Ciências pelo Programa de Pós-Graduação em Enfermagem da Universidade Federal de Pelotas (PPGEnf/UFPEL). \\ Membro Integrante do GEP-GERON/FURG/CNPq. <janainaamorim23@hotmail.com> \\ ${ }^{5}$ Enfermeira. Doutora em Enfermagem. Docente da Escola de Enfermagem (EEnf/FURG). Membro Integrante do GEP-GERON/FURG/CNPq. \\ <barbarataroucos@gmail.com> \\ bEnfermeira. Doutora em Enfermagem. Docente do PPGEnf/FURG. Vice-líder do GEP-GERON/FURG/CNPq. <pmarleneteda@yahoo.com.br>
}

INTRODUÇÃO: O Caderno de Atenção Básica, no 36 - Estratégias para o cuidado da pessoa com doença crônica - Diabetes Mellitus, do Ministério da Saúde, destaca a consulta de enfermagem como fundamental. Esta é realizada pelo enfermeiro por meio do Processo de Enfermagem (PE). OBJETIVO: Refletir acerca da operacionalização do PE no cuidado à pessoa idosa com DMT2. METODOLOGIA: Realizou-se reflexão teórica embasada em produções científicas. $\bigcirc$ eixo que sustentou essa reflexão concentrou-se na Lei 7.498/86, na Resolução no 272/2002 e no art. 3o da Resolução 358/2009, o qual dispõe sobre a SAE e a implementação do PE, do COFEN. RESULTADOS: O PE se dá em cinco etapas. A primeira se dá por meio do Histórico de Enfermagem, constituído por entrevista e exame físico. A segunda é seguida pelo estabelecimento dos Diagnósticos de Enfermagem, realizados a partir de julgamento clínico das respostas do indivíduo, família e da comunidade aos processos vitais ou aos problemas de saúde atuais ou potenciais. $\bigcirc$ estabelecimento dos diagnósticos possibilitará cuidados pertinentes. São definidos e classificados conforme a North American Nursing Diagnosis Association (NANDA). A terceira etapa do PE consiste no Planejamento da Assistência, que vai direcionar os cuidados de enfermagem para as necessidades do cliente, por meio do planejamento das intervenções que serão realizadas. Exige raciocínio clínico. Após, realiza-se a quarta etapa do PE, a Implementação da Assistência, com a realização das ações ou intervenções, como o processo educativo. A quinta etapa do PE é a Avaliação dos Resultados, com verificação de mudanças nas respostas da pessoa, família ou coletividade, com reajustes, novos diagnósticos e prescrição. CONCLUSÃO: Constata-se a relevância do PE para a organização do cuidado. Explicita-se a Consulta de Enfermagem, com operacionalização do PE, como ferramenta no cuidado à pessoa idosa com DMT2 e como meio de visibilidade profissional. 


\section{Dados epidemiológicos sobre o suicídio (CID 10, X60-X84) entre idosos do Sul do Brasil (2011-2016)}

\section{Ferdinando Agostinho', Karoline Monteiro de Oliveira Cardoso², Maria Luiza DÁvila Sandri', Ondina Almeida Resende ${ }^{4}$, Franciely dos Passos Pereira ${ }^{5}$, Karlla Kristinna Almeida Medeiros 6 , Camila Botelho Miguel ${ }^{7}$, Wellington Francisco Rodrigues ${ }^{8}$}

'Faculdade Morgana Potrich (FAMP)/Universidade do Rio Verde (UNIRV/Universidade Federal do Triângulo Mineiro (UFTM).<ferdinando@unirv.edu.br> 2FAMP.<karolineoliver2@hotmail.com>

${ }^{3}$ FAMP. < mldsandri@hotmail.com>

4FAMP. <ondina.a.resende@hotmail.com>

5 FAMP < pereirafrancielyp@gmail.com>

${ }^{6}$ FAMP/UNIRV/UFTM. < karllakristinna@hotmail.com>

${ }^{7}$ FAMP/UNIRV/UFTM. < camilabmiguel@hotmail.com>

${ }^{8}$ FAMP/UNIRV/UFTM. <wellington.frodrigues@hotmail.com>

INTRODUÇÃO: O suicídio é uma das principais causas de morte no mundo e um importante problema de saúde pública. Entre idosos, a principal causa de suicídio é a presença de depressão. OBJETIVOS: Este estudo objetivou descrever a taxa de incidência de casos de suicídios entre idosos na região sul do Brasil, estratificados por faixa etária e categoria do CID 10. MÉTODO: Foram utilizados dados do IBGE/CENSO2010 e SIM/Datasus do qual foram selecionadas as categorias do CID10 (X60-X84) no período de 2011 a 2016. A taxa de incidência (TI) foi calculada para duas faixas etárias: $\leq 59$ e $\geq 60$ anos, utilizando a fórmula (número de casos registrados no período x 10.000/população). RESULTADOS: Foram identificados 14.293 casos de suicídio, destes, aqueles envolvendo idosos representaram 22,27\% (3.184), neste grupo, a incidência de casos foi maior entre os homens $(81,50 \%)$ e a categoria do CID 10 com maior incidência de casos foi X70 "Lesao autoprovocada intencionalmente por enforcamento, estrangulamento ou sufocamento" (2.296 casos $(72,11 \%))$. A TI de mortes por suicídio na população em geral foi de 5,22 (p/ 10.000 habitantes (hab)); para idosos ( $\geq 60$ anos), a TI foi de 9,69 (p/10.000 hab) versus $4.60(\mathrm{p} / 10.000 \mathrm{hab})$ da faixa etária $\leq 59$ anos. Para idosos do sexo masculino a categoria com maior incidência de casos foi "X70" (335 casos (63,2\%)), a Tl foi de 17,78 (p/10.000 hab) versus 7,24 (p/10.000 hab) da faixa etária $\leq 59$ anos. Para as idosas a categoria do CID 10 com maior incidência de casos também foi "X70" (55 casos (50,92\%)), a TI foi de 3,22 (p/10.000 hab) contra 2,00 (p/10.000 hab) da faixa etária $\leq 59$ anos. CONCLUSÃO: Pode-se concluir que a taxa de incidência de mortes ocasionadas por suicídio entre idosos é maior que a de outras faixas etárias, possuindo características específicas entre os sexos. Evidencia-se que o suicídio é um problema de saúde pública e deve ser obrigatoriamente considerado em Gerontologia e Geriatria. 


\title{
Baixa mobilidade e desnutrição como preditores de mortalidade em pacientes idosos com Diabetes Melito tipo 2
}

\author{
Mileni Vanti Beretta', Juliane V. Feldman², Ticiana da Costa Rodrigues ${ }^{2}$ \\ 'Programa de Pós-Graduação em Ciências Médicas: Endocrinologia, Universidade Federal do Rio Grande do Sul (UFRGS). Porto Alegre, RS, Brasil. \\ <mberetta@hcpa.edu.br> (correspondente) \\ UUFRGS. Porto Alegre, RS, Brasil.
}

\begin{abstract}
INTRODUÇÃO: Em idosos com diabetes melito tipo 2 (DM2) parece existir uma forte correlação entre a marcha e piores desfechos como quedas, imobilização e mortalidade. OBJETIVO: determinar a relação entre o teste de caminhada e a mortalidade por todas as causas após alta hospitalar em idosos com DM2. METODOLOGIA: Estudo prospectivo que incluiu pacientes hospitalizados no Hospital de Clínicas de Porto Alegre (HCPA) entre julho de 2015 e dezembro de 2016, acima de 60 anos e com até 48 horas de internação. Para avaliar a mobilidade, realizou-se um teste de marcha, como Time Up and Go (TUG) considerando como baixa mobilidade $<0,8 \mathrm{~m} / \mathrm{s}$; e o estado nutricional pela Mini avaliação nutricional. Este projeto foi aprovado pelo Comitê de Ética do HCPA sob o número 150068. RESULTADOS: 312 pacientes com DM2 foram incluídos com as seguintes características: idade média de $71.31(6), 51 \%$ do sexo feminino, 82\% caucasianos. O TUG apresentou correlação positiva com a idade $(r=0,283, p<0,001)$, tempo de internação $(r=0,081, p=0,17)$ e correlação negativa com IMC $(r=-0,42, p=0,48)$ e HbAlc $(r=-0,06, p=0,91)$. A mortalidade no período foi de $20 \%$. Na análise multivariada, após ajustes, mantiveram-se associados à mortalidade: a desnutrição avaliada pelo $M N A$ e $T U G<0,8 \mathrm{~m} / \mathrm{s}(\mathrm{OR}=2.064,95 \% \mathrm{Cl}$ 1.048-4.063 p=0.036 e $\mathrm{OR}=0,872$, $95 \% \mathrm{Cl}$ 0,78-0,96 p=0,010). CONCLUSÃO: A presença de desnutrição e a baixa mobilidade foram preditores de mortalidade em pacientes com DM2. Ambas as medidas são de fácil aplicabilidade na rotina clínica podendo ser utilizadas como medidas de rastreio, sugerindo que a detecção precoce da redução da funcionalidade e a correta intervenção possa reduzir o risco de mortalidade.
\end{abstract}




\title{
Associação entre qualidade da dieta e sarcopenia em longevos
}

\section{Francine da Rocha Flores Giediel Rosa', Amanda Guterres Beuren², Roberta Rigo Dalla Corte ${ }^{2}$, Ticianada Costa Rodrigues ${ }^{3}$, Renato Bandeira de $\mathrm{Mello}^{3}$}

\author{
Programa de Pós-Graduação em Endocrinologia, Universidade Federal do Rio Grande do Sul (UFRGS). Porto Alegre, RS, Brasil. \\ <francinefflores@hotmail.com> (correspondente) \\ ${ }^{2}$ Faculdade de Medicina, UFRGS. Porto Alegre, RS, Brasil. \\ ${ }^{3}$ Programa de Pós-Graduação em Endocrinologia, Faculdade de Medicina, UFRGS. Porto Alegre, RS, Brasil.
}

INTRODUÇÃO: A faixa etária com idade igual ou superior a 80 anos - longevos - é o maior segmento populacional em crescimento. Dentre mudanças que ocorrem com o avançar da idade, a Sarcopenia caracteriza-se por perda involuntária de massa, força e qualidade muscular. OBJETIVO: Verificar a associação entre qualidade da dieta e sarcopenia em idosos longevos. MÉTODO: Estudo transversal. 40 longevos atendidos no ambulatório de geriatria do Hospital de Clínicas de Porto Alegre foram incluídos. O estado nutricional foi classificado pelo Índice de Massa Corporal (IMC) e o Índice de Alimentação Saudável pelo instrumento Healthy Eating Index - HEl através de recordatório de 24 horas. Composição corporal medida pela Bioimpedância (BIA). Performance muscular por velocidade de marcha em $4 \mathrm{~m}$. Para força, utilizou-se o dinamômetro de preensão palmar. RESULTADOS: A média de idade foi $83,5 \pm 2,81$ anos, sendo $60 \%$ da amostra representada pelo sexo feminino. $\bigcirc$ Índice de massa corporal médio foi de $27,35 \mathrm{~kg} / \mathrm{m}^{2}$. No total da amostra, $62,5 \%$ das idosas e 33,3 dos idosos apresentaram sarcopenia. Do total de sarcopênicos, 45,5\% dos homens e 83,3\% das mulheres tiveram baixa qualidade da dieta, com tendência a associação entre os dois fatores nesta análise preliminar. CONCLUSÃO: Dados preliminares de 40 idosos apontam para uma tendência de associação entre dieta e sarcopenia em longevos. A coleta de dados segue para um n estimado de 140 idosos. 


\title{
Diferenças de gênero no número de fatores de risco cardiovasculares modificáveis entre idosos jovens e longevos, PNS 2013
}

\author{
Fabiane de Oliveira Brauner', Renata Breda Martins², Josemara de Paula Rocha ${ }^{2}$, \\ Andressa Dode², Ângelo José Gonçalves Bós² \\ 'Pontifícia Universidade Católica do Rio Grande do Sul (PUCRS). Porto Alegre, RS, Brasil. <braunerpro@gmail.com> (correspondente) \\ ${ }^{2}$ PUCRS. Porto Alegre, RS, Brasil.
}

\begin{abstract}
INTRODUÇÃO: Doenças cardiovasculares (DCV) impactam na saúde dos idosos (60 a 79 anos) e longevos (80 anos e mais). Pouco se sabe sobre a frequência dos fatores de risco modificáveis (FRM) em idosos e longevos no Brasil. OBJETIVO: Observar diferenças de gênero no número de FRM entre idosos jovens e longevos. MÉTODOS: Análise secundária de dados da Pesquisa Nacional de Saúde, 2013. Os FRM foram: obesidade (índice de massa corporal $>27 \mathrm{~kg} / \mathrm{m}^{2}$ ), sedentarismo (<150minutos/ semana), tabagismo atual, consumo excessivo de álcool e sal, diagnóstico de hipertensão arterial sistêmica (HAS) e diabetes (DM). Foram calculadas as médias do número de FRM entre idosos e longevos e homens e mulheres e testadas pelo t-Student. Frequências de cada FRM foram calculadas e testadas pelo Qui-quadrado. RESULTADOS: Do total de 11.177 indivíduos, $86,6 \%$ eram idosos. A média de FRM foi significativamente maior nos idosos $(1,13 \pm 0,80)$ que nos longevos $(1,04 \pm 0,59$, $p=0,001)$. Os homens idosos apresentaram maior média de $\operatorname{FRM}(1,28 \pm 0,85)$ que os longevos $(1,04 \pm 0,64, p<0,001)$. Já para as mulheres as médias foram similares $(1,03 \pm 0,74$ e 1,05 00,56 respectivamente). Os FRM mais frequentes em idosos do que idosas foram: o consumo de álcool (9\%) e sal (10\%), fumo atual (18\%) e sedentarismo (64\%). Consumo de álcool (0,9\%) e fumo atual (9\%) também foram mais frequentes em homens longevos. As mulheres tanto idosas quanto longevos apresentaram com maior frequência HAS (54\%, 59\%), obesidade (45\% e 30\%) e DM (20\% e 19\%). As longevas apresentaram também maior consumo de sal (5\%) e sedentarismo (83\%). CONCLUSÕES: Homens idosos foram os que apresentaram maior número de FRM. A diferença na média de FRM foi também maior entre os homens idosos e longevos do que entre as idosas e longevas. Maior mortalidade por DCV pode ser a principal razão para as diferenças encontradas nos idosos mais jovens. Observa-se assim a necessidade de políticas que estimulem o controle de FRM em idosos homens.
\end{abstract}




\title{
A compreensão do fenômeno do envelhecimento em uma instituição de longa permanência
}

\author{
Margarita Ana Rubin Unicovsky', Ana Karina Silva da Rocha Tanaka ${ }^{2}$ \\ 'Escola de Enfermagem, Universidade Federal do Rio Grande do Sul (UFRGS).Porto Alegre, RS, Brasil. <mar.u@terra.com.br> \\ 2Escola de Enfermagem, UFRGS. Porto Alegre, RS, Brasil. <ana.tanaka@ufrgs.br>
}

INTRODUÇÃO: O corpo é compreendido como um conjunto de órgãos e funções, e a velhice, como as alterações que nele ocorrem. OBJETIVO: Esta investigação buscou compreender a concepção de envelhecimento no idoso em uma instituição de longa permanência. MÉTODO: $O$ método utilizado foi do tipo fenomenológico com abordagem qualitativa. A pesquisa foi realizada em uma instituição de longa permanência, de caráter privado, escolhida pela disponibilidade de acesso e por possuir uma filosofia voltada para a satisfação e para a melhoria de qualidade de vida dos residentes, com oito participantes. A coleta de informações empregada foi à entrevista dialógica, semi - estruturada, a partir de perguntas orientadoras, que oportunizaram o desvelamento da concepção de cada um dos participantes na caminhada para atingir a essência do fenômeno. Este processo foi permeado com o referencial teórico que nos possibilitou compreender, aprofundar e avançar no conhecimento das experiências significativas destes idosos, bem como nos auxiliou na compreensão da metodologia, na analise do material, na construção das dimensões e no desvelamento da essência fenomenológica. Esta pesquisa passou por todos os processos éticos institucionais CAAE: 0946/2003 e assinatura do termo de consentimento Livre e esclarecido. RESULTADOS: Na identificação e contextualização das concepções dos idosos sobre o corpo e envelhecimento, emergiram quatro dimensões fenomenológicas: o envelhecimento biológico, o psicológico, o social e o significado do corpo na velhice, como uma essência, o velho esse outro. Os resultados apontaram que o corpo é compreendido como um conjunto de órgãos e funções, e a velhice, como as alterações que nele ocorrem. CONCLUSÃO: O significado de corpo na velhice não está no que ele é, mas no que ele representa, ele exalta a vida e suas diversas probabilidades, mas ao mesmo tempo evidencia a sua finitude. 


\title{
A prevalência de Massa óssea e níveis de vitamina $D$ em indígenas idosos e de meia idade da Zona Rural do Sul do Brasil
}

\author{
Ana Karina Silva da Rocha Tanaka', Ângelo Bós², Denise Cantarelli Machado², \\ Margarita Ana Rubin Unicovsky ${ }^{3}$, Claudine Lamanna Schirmer ${ }^{2}$, Valéria Baccarin laniski²
}

'Escola de Enfermagem, Universidade Federal do Rio Grande do Sul (UFRGS). Porto Alegre, RS, Brasil. <ana.tanaka@ufrgs.br> (correspondente)

2 Programa de Pós-Graduação em Gerontologia Biomédica, PUCRS. Porto Alegre, RS, Brasil.

${ }^{3}$ Escola de Enfermagem, UFRGS. Porto Alegre, RS, Brasil.

INTRODUÇÃO: $O$ envelhecimento indígena brasileiro dispõe de dados globais insípidos sobre a situação de saúde da população, apenas dados parciais fornecidos pela FUNAI, FUNASA. As taxas de mortalidade e morbidade são de três a quatro vezes maiores que da população brasileira em geral. Em relação à massa óssea, quanto maior o pico atingido pelo indivíduo, maior será a sua reserva de cálcio para o período do envelhecimento e menor será a sua suscetibilidade a fraturas. OBJETIVO: A presente pesquisa teve por objetivo descrever a massa óssea e níveis de vitamina $D$ em indígenas de meia idade e idosos do meio rural do sul do Brasil. MÉTODO: Estudo transversal, descritivo e analítico. Participaram 73 indígenas com 40 anos ou mais do município de Nonoai RS, Brasil, etnia Kaingang. Os indígenas foram transportados até o hospital de grande Porte, para realização da pesquisa através da assinatura do TCLE. Foram realizadas dosagens de vitamina D. A densitometria óssea computadorizada foi realizada da região do fêmur proximal e da coluna lombar. Esta pesquisa foi aprovada pela CONEP e CNPq para verificação de mérito cientifico, CEP no 11/05323 CONEP, Parecer no 497/2011. RESULTADOS: A avaliação da massa óssea foi realizada por densitometria das regiões da coluna e fêmur. Em relação à massa óssea, observou-se que as principais alterações localizavam-se na coluna 46 (63\%). Com relação à massa óssea do fêmur, apenas 19\% dos indivíduos analisados apresentavam alterações. Em relação aos níveis séricos de vitamina D, observou-se que 49 (67, $1 \%$ ) estavam alterados. Em relação à frequência alimentar, houve uma associação entre massa óssea e níveis séricos de vitamina $D$ em relação a adição de sal nos alimentos. CONCLUSÃO: Acredita-se que a educação para a saúde dos indivíduos portadores de alteração de massa óssea seja o melhor caminho para o controle desse problema, desde que se promova a sua adequação para a cultura indígena e a motivação para mudanças de hábitos visando à melhora da qualidade de vida. 


\title{
Da hipertensão arterial à insuficiência renal crônica no idoso
}

\author{
Luciana de Almeida Cunha', Valéria Baccarin laniski², Carla Kowalski ${ }^{3}$, \\ Ibrahim Clós Mahmud ${ }^{4}$, José Roberto Goldim 5
}

\author{
'Aluna do curso de Doutorado, Programa de Pós-Graduação em Gerontologia Biomédica (GERONBIO), Escola de Medicina, \\ Pontifícia Universidade Católica do Rio Grande do Sul (EM/PUCRS). Bolsista CAPES/PROEX. \\ ${ }^{2}$ Aluna do curso de Mestrado, GERONBIO-EM/PUCRS. Bolsista CAPES/PROEX. \\ ${ }^{3}$ Aluna do curso de Doutorado, GERONBIO-EM/PUCRS. Bolsista CAPES/PROEX \\ ${ }^{4}$ Aluno do curso de Mestrado, GERONBIO-EM/PUCRS. Bolsista CAPES/PROEX. \\ ${ }^{5}$ Biólogo. Professor do GERONBIO-EM/PUCRS.
}

\begin{abstract}
INTRODUÇÃO: A insuficiência renal crônica (IRC) é um estágio irreversível da função renal, decorrente de falha no equilíbrio metabólico e hidroeletrolítico, muitas vezes relacionado a hipertensão arterial sistémica (HAS), necesitando de terapias substitutivas, como a diálise. OBJETIVO: Identificar as possíveis causas da doença já instalada, promovendo junto ao paciente uma sensibilização quanto a importância de hábitos saudáveis, como prática de atividade física e alimentação adequada ao seu quadro clínico. MÉTODOS: Relato de experiência acerca do cuidado ofertado em meio hospitalar à idoso com IRC. RESULTADOS: A.B.F.M., homem, casado, 74 anos, internou em um hospital geral com diagnóstico de IRC em virtude de uma HAS descompensada. Fazia uso de sete medicamentos concomitantemente, no entanto, alguns com a mesma finalidade. Seus exames laboratoriais demonstravam alterações séricas nos níveis de ureia e creatinina ( $88 \mathrm{mg} / \mathrm{dL}$ e 2,60 mg/dL, respectivamente), o que caracterizava função renal seriamente prejudicada. Ao exame físico constatou-se a presença de edema de grau 3 na região dos pés e calcâneo, xerostomia, desidratação e extremidades em condição à erupção de feridas. Foi realizada terapia substitutiva e ofertada dieta hipossódica (a qual teve baixa aceitação). Em posterior análise aos níveis séricos, foi orientado o paciente no momento da alta hospitalar, a importância da mudança de hábitos principalmente relacionados à alimentação e atividade física, evidenciando a gravidade da doença, sinais e sintomas e meios de controle. Conclusões: As recomendações interdisciplinares para alta hospitalar são estratégias importantes para a manutenção da saúde e equilibrio das doenças crônicas para a não agudização e cronificação.
\end{abstract}




\title{
Leucina e síntese de proteínas musculares em idosos: uma revisão integrativa
}

\author{
Vitor Buss', Valéria Baccarin laniski², Luana Fioravanti Roland ${ }^{3}$, Evelise Moraes Berlezi ${ }^{4}$ \\ 'Nutricionista. Mestrando do Programa de Pós-Graduação em Gerontologia, Universidade Federal de Santa Maria (UFSM). \\ Santa Maria, RS, Brasil. < vitor.buss@hotmail.com> \\ ${ }^{2}$ Nutricionista. Mestranda do Programa de Pós-Graduação em Gerontologia Biomédica, Pontifícia Universidade Católica do Rio Grande do Sul (PUCRS). \\ <valeriaianiski@yahoo.com.br> \\ 3Nutricionista. Mestranda do Programa de Pós-Graduação em Gerontologia, UFSM. Santa Maria, RS, Brasil. <luanafioravanti.ufsm@gmail.com> \\ ${ }^{4}$ Fisioterapeuta. Professora titular da Universidade Regional do Noroeste do Estado do Rio Grande do Sul (UNIJUÍ) e professora convidada do \\ Programa de Pós-Graduação em Gerontologia, UFSM. <evelise@unijui.edu.br>
}

\begin{abstract}
INTRODUÇÃO: No envelhecimento patológico a incapacidade funcional é frequentemente observada e impacta no maior risco de dependência, sendo esta condição relacionada sobretudo com o componente físico, a exemplo da redução de massa muscular. Concomitantemente, é reconhecido o papel dos aminoácidos essenciais, em especial a leucina, na síntese de proteínas musculares. OBJETIVO: Elucidar o papel da suplementação de leucina na síntese de proteínas musculares em idosos. METODOLOGIA: Revisão bibliográfica de ensaios clínicos com idosos, publicados nos últimos 5 anos e indexados na plataforma PUBMED, sendo que a busca ocorreu com os descritores "leucine", "muscle protein synthesis" e "aging". RESULTADOS: Foram encontrados 20 artigos, dos quais 6 foram excluídos por não abordarem o foco da pesquisa. Na análise do restante observou-se efeito positivo da suplementação de leucina na síntese de proteínas musculares em 9 (64\%) artigos, sendo que a dose mínima utilizada foi de $1.2 \mathrm{~g}$ e a máxima 13,2 g de leucina/dia, com uma tendência ao melhor aproveitamento nos intervalos das principais refeições, sem inferência de resultados positivos na melhora da força e/ou função muscular. Além disso, 7 (50\%) artigos foram conduzidos com homens e mulheres. Entre as limitações destacam-se o financiamento de alguns estudos por indústrias de suplementos nutricionais, o tamanho amostral, seleção prioritária de indivíduos saudáveis e o curto tempo de duração. CONCLUSÕES: Fica evidente o potencial benefício da suplementação de leucina para estímulo da síntese de proteínas musculares em idosos, no entanto, os resultados não são conclusivos para indivíduos com depleção muscular, por isso, sugere-se que estudos complementares preencham esta lacuna e estabeleçam a recomendação de dose/resposta ou tempo de tratamento para maior efetividade.
\end{abstract}




\section{O cuidador de idosos como paciente: quem cuida de quem cuida?}

\section{Caroline Sbardellotto Cagliari' ${ }^{1}$ Bruna Tiemi Onishi Ogliari², Laís Valiati Boff ${ }^{3}$, Naiara Degrande ${ }^{4}$, Rafaely Guinzelli5 , Débora da Luz Becker ${ }^{6}$, Dener Lizot Rech7 ${ }^{7}$, Roberto Luis Bigarella ${ }^{8}$}

'Acadêmica do Curso de Medicina da Universidade de Caxias do Sul (UCS). Caxias do Sul, RS, Brasil. <sccaroline@hotmail.com>

${ }^{2}$ Acadêmica do Curso de Medicina da UCS. Caxias do Sul, RS, Brasil. < bruna.tiemi@hotmail.com>

${ }^{3}$ Acadêmica do Curso de Medicina da UCS. Caxias do Sul, RS, Brasil. < lais.valiati@hotmail.com>

${ }^{4}$ Acadêmica do Curso de Medicina da UCS. Caxias do Sul, RS, Brasil. <naia.degrande@gmail.com>

${ }^{5}$ Acadêmica do Curso de Medicina da UCS. Caxias do Sul,RS, Brasil.<rfy@hotmail.com>

${ }^{\circ}$ Acadêmica do Curso de Medicina da UCS. Caxias do Sul, RS, Brasil. < de dabecker@hotmail.com>

7Docente da Disciplina de Geriatria do Curso de Medicina da UCS. Caxias do Sul, RS, Brasil. <dlrech1@ucs.br>

${ }^{8}$ Docente da Disciplina de Geriatria do Curso de Medicina da UCS. Caxias do Sul, RS, Brasil. <rlbigarella1@ucs.br>

INTRODUÇÃO: $\bigcirc$ envelhecimento da população acarreta mais cuidados para os idosos, uma vez que $85 \%$ possuem pelo menos uma doença crônica. Com isso, a responsabilidade sobre o paciente pode aumentar e ultrapassar limites psicofísicos do cuidador, gerando adoecimento. É essencial compreender que o cuidador também necessita de atenção. OBJETIVOS: Realizar pesquisa acerca da atenção dada aos cuidadores de idosos. MÉTODOS: Estudo transversal com revisão bibliográfica de artigos sobre o cuidado ao cuidador do idoso nas bases de dados Pubmed e Scielo, utilizando os termos "eldery's caregivers", "caregiver" e "quality of life", nos últimos 10 anos. RESULTADOS: O aumento de idosos no Brasil foi de 18\%, sendo o Rio Grande do Sul um dos 2 estados com maior representatividade de 2012 a 2017 , segundo o IBGE. O aumento também ocorre com doenças crônicas sendo a participação de cuidadores na vida do paciente idoso fundamental. Percebe-se que cuidados integrais acabam por afetar negativamente na qualidade de vida da pessoa cuidadora do idoso e, por isso, busca-se a importância de dar mais atenção a essas pessoas para evitar o seu esgotamento. Fornecer informações detalhadas ao cuidador é o o ajuda a prover o melhor cuidado. Além disso, a compreensão da multidisciplinariedade é essencial por envolver uma boa relação entre todos os envolvidos. Entretanto, não há um enfoque tão relevante sobre à inclusão dos cuidadores no posto de pacientes e muito menos em proporcionar alternativas para cuidar de quem cuida. CONCLUSÃO: É sabido a incidência sobre estresse do cuidador, sendo necessário enfoque em estratégias para combater tal sobrecarga. Essas alternativas, porém, não são bem elaboradas nos artigos revisados. Observou-se a falta de reflexão sobre quem cuida de quem cuida apesar de a relação multidisciplinar e o acesso à informação por parte dos cuidadores ser alvo de enfoque. Se é necessário um cuidado eficaz com os idosos, é necessário também um cuidado eficaz dos seus cuidadores. 


\title{
Aprendizagem digital: um novo espaço de inclusão social para os idosos
}

\author{
Tatiana Benini', Vania Beatriz Merlotti Herédia', Verônica Bohm³, Délcio Antônio Agliardi ${ }^{4}$ \\ Universidade de Caxias do Sul (UCS). Caxias do Sul, RS, Brasil. <tbenini@ucs.com> \\ 2 UCS. Caxias do Sul, RS, Brasil. < vbmhered@gmail.com> \\ ${ }^{3}$ UCS. Caxias do Sul, RS, Brasil.<vebohm@bol.com.br> \\ ${ }^{4}$ UCS. Caxias do Sul, RS, Brasil. <daagliar@ucs.com>
}

\begin{abstract}
INTRODUÇÃO: A inclusão social de idosos em atividades atuais que preveem o uso de tecnologias digitais implica na apropriação desses recursos que não são de conhecimento de muitos idosos. OBJETIVO: $\bigcirc$ presente estudo analisa a experiência realizada por alunos que frequentam o Programa UCS Sênior da Universidade de Caxias do Sul, em disciplinas que abordam aprendizagens digitais e tem como objetivo verificar como se dá o processo de aprendizagem dessas novas tecnologias e os mecanismos utilizados que facilitam ou dificultam o processo. MÉTODO: O estudo, de natureza exploratória faz uso do método descritivo com a pretensão de contribuir para a compreensão da inserção dessa população no mundo digital. A pesquisa foi dividida em duas etapas: a primeira, a realização de uma pesquisa empírica, quantitativa, constituída por 428 sujeitos, sendo 357 mulheres e 71 homens cujo objeto investiga como se dá o processo de aprendizagem, de forma objetiva. A segunda etapa, de natureza qualitativa, foi realizada por meio de entrevista semiestruturada com esses idosos, a fim de indagar sobre aspectos subjetivos do processo de aprendizagem. RESULTADOS: Os resultados apontam que um dos principais motivos para a busca de conhecimento em tecnologias digitais foi acompanhar a evolução das tecnologias digitais para estabelecer comunicação com os familiares, parentes, amigos e redes sociais. Os entrevistados afirmam que aprendem com a presença do professor e que as oficinas oferecidas são momentos de convivência, cumplicidade e aprendizagem. CONCLUSÕES: Os cursos frequentados acerca de tecnologias digitais proporcionam, além do aprendizado, uma forma de inclusão social, que facilita e amplia o contato dos idosos com suas famílias, amigos e inclusive atendem às exigências de serviços na sociedade contemporânea.
\end{abstract}




\title{
Consumo de benzodiazepínicos na atenção básica à saúde em um dos municípios com maior percentual de população idosa do Brasil
}

\author{
Daniel Capalonga', Juliana da Rosa Wendt²
}

'Universidade Federal de Santa Maria (UFSM). Santa Maria, RS, Brasil. < danielcapalonga@gmail.com>

2UFSM. Santa Maria, RS, Brasil. <ju wendt@hotmail.com>

\begin{abstract}
INTRODUÇÃO: Os benzodizepínicos (BZD) estão entre os fármacos mais prescritos ao redor do mundo. O uso prolongado, mesmo em baixas dosagens é capaz de acarretar efeitos adversos, sobremaneira se administrados a pacientes idosos. $\bigcirc$ presente trabalho ocupou-se de analisar a dispensação de BZD, pelo serviço público de saúde, a população de uma cidade do interior do Rio Grande do Sul. Tal município foi escolhido por suas peculiaridades: trata-se de um dos 5 municípios com maior percentual de população idosa no país, consoante dados do CENSO, e acrescenta-se o fato de toda dispensação ser centralizada na única unidade de saúde pública do município. OBJETIVOS: O objetivo do presente trabalho foi avaliar a dispensação dos BZD pela atenção básica em um município com elevada proporção de idosos no Rio Grande do Sul, analisando quais os fármacos ofertados, e o consumo dos BZD pelo público em estudo. MÉTODOS: Trata-se de um estudo no qual foi realizado um diagnóstico situacional e uma revisão da literatura científica para embasar uma discussão acerca dos dados encontrados. Esse é um estudo transversal, do tipo quantitativo descritivo, observacional, a partir da análise de dados secundários disponíveis a partir do Software Multi24 utilizado na referida unidade para gerenciamento farmacêutico. RESULTADOS: O levantamento foi realizado compreende o período de 02/01/2018 a 01/04/2018, permitiu identificar que atualmente os BZD fornecidos na unidade são o Clonazepam $2 \mathrm{mg}$ em comprimidos e também Clonazepam 2,5 mg/mL, solução oral, gotas. No período foram dispensados 2380 comprimidos, a um total de 23 pacientes, sendo 12 idosos; essa faixa populacional consumiu no período 1200 comprimidos, cerca de 50\% do total dispensado. Quanto a solução oral, foram dispensados 36 frascos a 17 pacientes sendo 10 idosos, que consumiram 19 frascos. CONCLUSÃO: O consumo de BZD por idosos é uma realidade, o achado nessa pesquisa confirma o fato. Na unidade pesquisada os idosos representam cerca de $50 \%$ dos usuários desses fármacos. A prescrição adequada é imperiosa, quando indispensável, devem-se ter claras as particularidades quanto ao seu uso em idosos em decorrência das alterações metabólicas oriundas do processo de envelhecimento, bem como o risco aumentado de efeitos adversos nessa faixa etária.
\end{abstract}




\title{
Avaliação cognitiva em idosos institucionalizados: uma análise comparativa
}

\author{
Caroline de Miranda', Letícia Brancher², Aniuska Schiavo³, Eléia de Macedo ${ }^{4}$ \\ 'Universidade de Caxias do Sul (UCS). Caxias do Sul, RS, Brasil.<carol miranda87@hotmail.com> \\ 2 UCS. Caxias do Sul, RS, Brasil. <letibrancher88@gmail.com> \\ ${ }^{3}$ UCS. Caxias do Sul, RS, Brasil. <aniuskaschiavo@hotmail.com> \\ ${ }^{4}$ UCS. Caxias do Sul, RS, Brasil. <eleiamacedo@yahoo.com.br>
}

INTRODUÇÃO: $\bigcirc$ envelhecimento populacional ocorre segundo mudanças sociais, culturais e econômicas, que modificam a configuração dos arranjos familiares. As deficiências cognitivas (DC) são as principais causas de institucionalização de idosos. A avaliação multidisciplinar e o planejamento de condutas contribuem para detecção precoce das deficiências cognitivas. OBJETIVO: Avaliar e comparar a condição cognitiva de idosos de instituição filantrópica e privada, a fim de verificar a relação entre gênero, idade, escolaridade, tempo de institucionalização e condição socioeconômica. MÉTODOS: Trata-se de um estudo do tipo transversal. Onde foram avaliados 16 idosos institucionalizados de ambos os gêneros, sendo 8 residentes de instituição filantrópica e 8 da privada, em Caxias do Sul/RS. Os idosos foram submetidos ao Mini Exame do Estado Mental (MEEM) e o Teste do Desenho do Relógio (TDR). RESULTADOS: Não se obteve resultados estatisticamente significativo entre os testes e as variáveis conforme o Teste Mann-Whitney com significância de 5\% ( $\leq \leq 0,05)$. Ao analisar as médias dos testes pode-se perceber que as variáveis interferiram no resultado dos testes, onde o melhor desempenho foi obtido pelos idosos de instituição privada. CONCLUSÃO: Sugere-se que as variáveis como idade e condição socioeconômica/cultural podem interferir no estado cognitivo dos idosos, demostrando que idosos com renda apresentam melhor estado cognitivo. 


\title{
Atenção domiciliar com idosos: o médico no âmbito da atenção básica
}

\author{
Bruna Thaise Lavagnini², Ibrahim Mahmud ${ }^{2}$, Carla Viero Kowalski ${ }^{3}$ \\ 'Médica. Especialista em Saúde da Família, UFCSPA. Porto Alegre, RS, Brasil. <bruna lavagnini@hotmail.com> \\ ${ }^{2}$ Médico. Especialista em Saúde da Família, UFCSPA. Especializando em Geriatria Clínica, FMUP/POR. Mestrando em Gerontologia Biomédica, \\ IGG/PUCRS. Bolsista CAPES/PROEX.Porto Alegre, RS, Brasil. <ibrahim mahmud@hotmail.com> \\ ${ }^{3}$ Enfermeira. Mestre em Saúde Coletiva, UNISINOS. Doutoranda em Gerontologia Biomédica, IGG/PUCRS. Bolsista CAPES/PROEX. \\ Porto Alegre, RS, Brasil. <arlakowalski25@gmail.com>
}

INTRODUÇÃO: O cuidado domiciliar à saúde é uma prática que remonta a própria existência da família como unidade de organização social. No Brasil a assistência domiciliar abrange diversos aspectos, ações de visitas, atendimentos e internações domiciliares. A atenção domiciliar surge a partir da equipe da estratégia de saúde da família (ESF) e através de programas específicos como o "Melhor em Casa", sempre de acordo com o tipo de demanda do paciente. OBJETIVO: Analisar a produção científica da atuação do médico na visita domiciliar com idosos na Atenção Básica. METODOLOGIA: Revisão integrativa da literatura cientifica, usando a ferramenta de busca OMNIS (PUCRS), utilizando os descritores: "Visita domiciliar" e Saúde do idoso", foram inclusos artigos dos últimos 10 anos, revisados por pares e disponíveis nos seguintes idiomas: inglês, português e espanhol. RESULTADOS PARCIAIS: Na primeira parte do estudo foi realizada uma busca em que foram encontrados 139 artigos. Em uma leitura preliminar, apenas 22 estão relacionados diretamente com a temática em estudo, porém 12 artigos da área da enfermagem também trouxeram o papel do médico nesse contexto. CONCLUSÕES: Foi encontrada escassa literatura que trouxe o papel específico do médico durante a visita domiciliar ao paciente idoso no âmbito da atenção básica. Durante a leitura dos artigos emergiram três aspectos principais, a visita domiciliar como um espaço que permita construção de estratégias e ações, identificou-se diversos papéis do médico durante a ação e emergiram variados sentimentos por parte do mesmo. 


\section{Trabalho e envelhecimento}

\section{Amanda Bifano Balbinot ${ }^{1}$}

'Faculdade Luterana São Marcos. <ambalbinot@gmail.com>

INTRODUÇÃO: $\bigcirc$ envelhecimento da população brasileira vem acendendo, a expectativa de vida aumentando, as políticas e regras para a concessão de aposentadorias sendo revistas e este quadro abre o questionamento sobre, qual a realidade de trabalho para da população idosa no Brasil. OBJETIVOS: Este estudo tem por objetivo avaliar a participação de idosos dentro do quadro funcional de empresas, as atividades desempenhadas e como as empresas compreendem o envelhecimento dos profissionais. MÉTODO: Foi realizado um de estudo de casos múltiplos, sendo investigadas 6 empresas, com área de atuação distintas de Porto Alegre e da Grande Porto Alegre/RS. A pesquisa realizada é qualitativa e quantitativa e apresenta dados preliminares. RESULTADOS: Da análise, verifica-se aspectos como o número de profissionais acima dos 60 anos que compõem o total funcionários das empresas, verifica-se a media 3,5\% nesta faixa etária, quanto a ocupação destes, destaca-se principalmente, 55\% destes atuando em cargos de liderança, 25\% em docência. Em algumas empresas, constata-se a presença do fenômeno de etarismo', por meio de comentários associados ao envelhecimento, os quais podem se transformar em barreiras para os profissionais mais velhos, quanto a sua permanência e oportunidade profissionais dentro da empresa. $\bigcirc$ que nem sempre é percebido pelos funcionários que acabam sofrendo ou praticando. CONCLUSÕES: Os resultados apontam para a necessidade de intervenções unificadas, na medida em que o envelhecimento da força de trabalho torna-se um desafio para as organizações, sociedade e governo, com ações voltadas para políticas públicas. Pois, o número de funcionários idosos é efêmero, no tocante do quadro funcional das empresas e como esta população vem crescendo, deve-se trabalhar na criação de oportunidade, para acompanharem esta projeção.

1 Preconceito ou discriminação contra ou a favor de um grupo etário. 


\title{
O HIV em idosos: atuação do médico no âmbito da atenção básica
}

\author{
Ibrahim Clós Mahmud', Luciana de Almeida², Bruna Thaíse Lavagnini ${ }^{3}$, \\ Paulo Renato Petersen Behar ${ }^{4}$, Newton Luiz Terra ${ }^{5}$
}

'Médico. Especialista em Saúde da Família, UFCSPA. Especializando em Geriatria Clínica, FMUP/POR. Mestrando em Gerontologia Biomédica, IGG/PUCRS. Bolsista CAPES/PROEX. Porto Alegre, RS, Brasil. <ibrahim_mahmud@hotmail.com>

2 Enfermeira. Mestre em Envelhecimento Humano, UPF. Doutoranda em Gerontologia Biomédica, IGG/PUCRS. Bolsista CAPES/PROEX. Porto Alegre, RS, Brasil. ${ }^{3}$ Médica. Especialista em Saúde da Família, UFCSPA. Porto Alegre, RS, Brasil. <bruna lavagnini@hotmail.com>

${ }^{4}$ Infectologista. Doutor em Medicina. Docente na UFCSPA e Chefe do Departamento de Infectologia do Complexo Hospitalar Santa Casa de Misericórdia de Porto Alegre, RS, Brasil.

${ }^{5}$ Geriatra. Doutor em Gerontologia Biomédica. Diretor do Instituto de Geriatria e Gerontologia e Docente na PUCRS. Porto Alegre, RS, Brasil.

\begin{abstract}
INTRODUÇÃO: $O$ envelhecimento da população brasileira vem incrementando-se nos últimos anos, se estima que em 2025 o Brasil terá aproximadamente 30 milhões de pessoas com idade igual ou superior a 60 anos. Dados de 2015, do Departamento de HIV/AIDS da Secretária Estadual de Saúde do Rio Grande do Sul, trazem Porto Alegre como segunda cidade no Ranking do estado com uma taxa de detecção para AIDS de 74 casos para cada 100.000 habitantes e demonstrou-se que os casos notificados de infecção pelo HIV em maiores de 55 anos cresceram muito, quando em 2007 foram somente 19 e no ano de 2015 foram 295 casos confirmados. OBJETIVO: Analisar a produção científica da atuação dos médicos com pacientes idosos portadores de HIV no âmbito da Atenção Básica. METODOLOGIA: revisão integrativa da literatura científica, usando a ferramenta de busca OMNIS (PUCRS), utilizando os descritores: "HIV" e "Saúde do idoso" OR "Idosos", foram inclusos artigos dos últimos 5 anos, revisados por pares e disponíveis nos seguintes idiomas: inglês, português e espanhol. RESULTADOS: Em um primeiro momento encontrou-se 514 artigos, após a leitura dos resumos, foram selecionados 134 trabalhos e logo após a leitura completa, foram analisados os 28 artigos que apresentaram a discussão da temática proposta. CONCLUSÖES: Os médicos da atenção primária, em diversas situações, apresentam uma percepção defasada quanto a problemática do HIV/AIDS no cenário atual, pois a invisibilidade da sexualidade do idoso potencializa situações de risco durante o manejo dos mesmos levando a um diagnóstico tardio. Diversos fatores potencializam essa problemática, tais como a deficiência na prevenção primária e secundária nessa faixa etária e o aumento da expectativa de vida acompanhada do prolongamento de sua vida sexual ativa.
\end{abstract}

